\title{
GPR97-mediated PAR2 transactivation via a mPR3- associated macromolecular complex induces inflammatory activation of human neutrophils
}

Hsi-Hsien Lin ( $\boldsymbol{D}$ hhlin@mail.cgu.edu.tw )

Chang Gung University

Tai-Ying Chu

Céline Zheng-Gérard

University of Oxford https://orcid.org/0000-0002-8044-9371

Kuan-Yeh Huang

Chang Gung University

Yu-Chi Chang

Chang Gung University

Ying-Wen Chen

Chang Gung University

Kuan-Yu I

Chang Gung University

Yu-Ling Lo

Chang Gung University

Nien-Yi Chiang

University of Oxford

Hsin-Yi Chen

Chang Gung University

Martin Stacey

University of Leeds

Siamon Gordon

Sir William Dunn Schl of Pathology

Wen-Yi Tseng

Chang Gung Memorial Hospital-Keelung

Chiao-Yin Sun

Chang Gung Memorial Hospital-Keelung

Yen-Mu Wu

Chang Gung University

Yi-Shin Pan

Chang Gung Memorial Hospital-Linkou 


\section{Chien-Hao Huang}

Chang-Gung Memorial Hospital, Linkou Medical Center

\section{Chun-Yen Lin}

Department of Gastroenterology and Hepatology

\section{Tse-Ching Chen}

Chang Gung Memorial Hospital

\section{Marilina Antonelou}

University College London

\section{Scott Henderson}

University College London

\section{Alan salama}

UCL Department of Renal Medicine https://orcid.org/0000-0002-9255-9092

\section{Elena Seiradake}

Oxford University

\section{Article}

Keywords: adhesion GPCR, GPCR transactivation, inflammation, neutrophils

Posted Date: February 25th, 2022

DOI: https://doi.org/10.21203/rs.3.rs-1371330/v1

License: (c) (i) This work is licensed under a Creative Commons Attribution 4.0 International License. Read Full License

Version of Record: A version of this preprint was published at Nature Communications on October 27th, 2022. See the published version at https://doi.org/10.1038/s41467-022-34083-1. 
GPR97-mediated PAR2 transactivation via a mPR3-associated macromolecular complex induces inflammatory activation of human neutrophils

Tai-Ying Chu ${ }^{1,13}$, Céline Zheng-Gérard ${ }^{2,13}$, Kuan-Yeh Huang ${ }^{1}$, Yu-Chi Chang ${ }^{1}$, Ying-Wen Chen $^{1}$, Kuan-Yu I ${ }^{1}$, Yu-Ling Lo ${ }^{1}$, Nien-Yi Chiang ${ }^{1}$, Hsin-Yi Chen ${ }^{1}$, Martin Stacey ${ }^{3}$, Siamon Gordon $^{1,4}$, Wen-Yi Tseng ${ }^{5}$, Chiao-Yin Sun ${ }^{6,7}$, Yen-Mu Wu ${ }^{8,9}$, Yi-Shin Pan ${ }^{10}$, Chien-Hao Huang ${ }^{10}$, Chun-Yen Lin ${ }^{10}$, Tse-Ching Chen ${ }^{11}$, Marilina Antonelou ${ }^{12}$, Scott R Henderson ${ }^{12}$, Alan Salama ${ }^{12}$, Elena Seiradake ${ }^{2, *}$, and Hsi-Hsien $\operatorname{Lin}^{1,5,11,14, *}$

${ }^{1}$ Department of Microbiology and Immunology, College of Medicine, Chang Gung University, Taoyuan 33302, Taiwan.

2Department of Biochemistry, University of Oxford, Oxford, OX1 3QU, UK.

${ }^{3}$ Faculty of Biological Sciences, School of Molecular and Cellular Biology, University of Leeds, Leeds LS2 9JT, UK.

${ }^{4}$ Sir William Dunn School of Pathology, University of Oxford, Oxford, OX1 3RE, UK

${ }^{5}$ Division of Rheumatology, Allergy and Immunology, Chang Gung Memorial HospitalKeelung, Keelung 20401, Taiwan

${ }^{6}$ Department of Nephrology, Chang Gung Memorial Hospital-Keelung, Keelung 20401, Taiwan

${ }^{7}$ Department of Medicine, College of Medicine, Chang Gung University, Taoyuan 33302, Taiwan

${ }^{8}$ Graduate Institute of Clinical Medical Sciences, College of Medicine, Chang Gung University, Taoyuan 33302, Taiwan

${ }^{9}$ Division of Infectious Diseases, Department of Internal Medicine, Chang Gung Memorial Hospital-Linkou, Taoyuan 33305, Taiwan

${ }^{10}$ Department of Gastroenterology and Hepatology, Chang Gung Memorial HospitalLinkou, Taoyuan 33305, Taiwan

${ }^{11}$ Department of Anatomic Pathology, Chang Gung Memorial Hospital-Linkou, Taoyuan 33305, Taiwan

${ }^{12}$ Department of Renal Medicine, Royal Free Campus, UCL, Rowland Hill Street, London, NW3 2PF, UK

${ }^{13}$ These authors contributed equally

${ }^{14}$ Lead contact

*Correspondence: elena.seiradake@bioch.ox.ac.uk (E.S.), hhlin@mail.cgu.edu.tw (H.H.L.) 
Running title: GPR97 transactivates PAR2 in neutrophils via mPR3

Keywords: adhesion GPCR, GPCR transactivation, inflammation, neutrophils

Manuscript length: 35 pages (14 words in the Title, 146 words in the Abstract, 4,075 words in the main text, 3,664 words in Methods), 70 references, 7 figures, and 11 supplemental items (4 supplemental tables and 7 supplemental figures) 


\begin{abstract}
Neutrophils play essential anti-microbial and inflammatory roles in host defense, however their activities are tightly regulated as neutrophil dysfunction often leads to detrimental inflammatory and autoimmune diseases. Here, we identified a novel PR3/CD177/GPR97/PAR2/CD16b interactome as a critical modulator of neutrophil activation. Using multiple approaches, we deorphanized GPR97, a human neutrophilrestricted adhesion $\mathrm{G}$ protein-coupled receptor (aGPCR), as the interacting protein and allosteric activator of CD177-associated membrane proteinase 3 (mPR3). Structural and deletion analysis of the GPR97 extracellular region disclosed two independent mPR3-binding domains. The efficient binding and activation of mPR3 by GPR97 required a macromolecular CD177/GPR97/PAR2/CD16b interactome and resulted in the transactivation of PAR2, another GPCR. GPR97-mediated PAR2 transactivation in neutrophils elicited strong inflammatory activation, triggering anti-microbial activities and endothelial dysfunction. Altogether, we identify a novel aGPCR-GPCR transactivation mechanism that directs neutrophil activation and inflammation. The PR3/CD177/GPR97/PAR2/CD16b interactome represents a potential therapeutic target for neutrophil-mediated inflammatory diseases.
\end{abstract}




\section{INTRODUCTION}

Neutrophils (N $\mathrm{s}$ ) express a plethora of immune effector proteins including proteases, cytokines, and receptors which contribute significantly to both the innate and adaptive immune responses ${ }^{1}$. However, these $N \varphi$-derived molecules warrant stringent regulation as their uncontrolled activities are often associated with detrimental inflammatory and autoimmune diseases ${ }^{2}$. N $\varphi$ serine proteases (NSPs) are azurophilic granule proteins which play critical roles in anti-microbial and inflammatory responses ${ }^{3,}$ 4. Proteinase 3 (PR3) is unique among NSPs as it also represents the designated autoantigen ( $\mathrm{Ag}$ ) of granulomatosis with polyangiitis (GPA), an autoimmune disorder hallmarked by the inflammatory necrosis of small/medium-sized blood vessels and the common presence of anti-neutrophil cytoplasmic antibodies (ANCA) against PR3 5 . Interestingly, a fraction of PR3 is exocytosed and tethered to the N $\varphi$ membrane (mPR3) mainly by specific binding to the glycosylphosphatidylinositol (GPI)-anchored CD1776. Increased plasma PR3 and mPR3 levels are positively associated with GPA pathogenesis, however, the normal cellular function of mPR3 remains enigmatic ${ }^{7,8}$.

G protein-coupled receptors (GPCRs) are critically involved in the activation and chemotaxis of $\mathrm{N} \varphi s$, and deregulated GPCR activities in N $\varphi s$ usually result in immune dysfunction and clinical disease ${ }^{9}$. Protease-activated receptors (PARs) represent a group of unique GPCRs elicited by a tethered peptide ligand following the proteolytic shedding of their $\mathrm{N}$-terminal region ${ }^{10}$. PAR2 is the predominant PAR expressed by $\mathrm{N} \varphi s$, and its activation is involved in tissue inflammation and prenatal death associated with the autoimmune antiphospholipid syndrome (APS) ${ }^{11}$ 12. Trypsin is the prototypic activator of PAR2, however, many other proteinases are similarly able to activate or disarm PAR $2^{13}$. Intriguingly, PAR2 can also be transactivated by PAR1 due to the formation of PAR1-PAR2 heterodimer ${ }^{14}$. To date, it is not fully understood how PAR2 activation is triggered in human N $\varphi s$ and whether PAR2 transactivation occurs in N $\varphi s$. Adhesion GPCRs (aGPCRs) are atypical GPCRs by having a large extracellular region (ECR) before the seven-transmembrane (7TM) domain ${ }^{15}$. Most aGPCRs are expressed as a non-covalent bipartite complex due to the auto-proteolytic cleavage of ECR at the GPCR proteolysis site (GPS) within the GPCR autoproteolysis-inducing (GAIN) domain ${ }^{15}$. Consequently, many aGPCRs are activated by a tethered agonism mechanism remarkably similar to that of PARs which involves the unmasking of an internal agonistic peptide following the dissociation/dislocation of ECR upon binding to its cellular ligand(s) ${ }^{16}$. Human $\mathrm{N} \varphi s$ express several aGPCRs including EMR2/ADGRE2, 
EMR3/ADGRE3, CD97/ADGRE5, and GPR97/ADGRG3 ${ }^{17,18}$. Although an anti-microbial role has been demonstrated for GPR97 previously, the lack of specific ligands has hindered the mechanistic understanding of its function ${ }^{18}$. Here, we show that GPR97 is the binding partner and allosteric activator of MPR3 through the macromolecular CD177/GPR97/PAR2/CD16b receptor interactome. Intriguingly, GPR97-augmented mPR3 enzymatic activity cleaves and activates PAR2 leading to robust $N \varphi$ activation. In conclusion, our results uncover a novel aGPCR-GPCR transactivation mechanism in human $\mathrm{N} \varphi s$ that contributes to inflammatory responses.

\section{RESULTS}

Activated neutrophils up-regulate GPR97 expression which correlates with the inflammation and/or disease status of various inflammatory disorders

Up-regulated GPR97 transcript and protein have been identified in blood N $\varphi s$ and tissue-infiltrating N $\varphi s$ (TIN $\varphi s$ ) of diverse inflammatory disorders ${ }^{18}$. To further correlate the relationship of $\mathrm{N} \varphi$ GPR97 expression and tissue inflammatory status, we compared GPR97 expression in early- and late-stage appendicitis. Consistently, specific GPR97 expression was detected in TINQS which were more numerous in the late-stage appendicitis (Fig. 1a, b). Moreover, much higher GPR97 staining intensities were identified in the late-stage than the early-stage appendicitis (Fig. 1c).

Similarly, higher surface GPR97 levels were detected in blood N $\varphi s$ of bacterial sepsis patients than those of healthy controls $(\mathrm{HC})$ by flow cytometry analyses (Fig. 1d). Thereafter, we enrolled patients of GPA and microscopic polyangiitis (MPA), two distinct autoimmune diseases characterized by ANCA mostly directed toward PR3 and myeloperoxidase (MPO), respectively ${ }^{5}$. No significant differences of GPR97 expression were observed among $\mathrm{HC}$ and patients (Fig. 1e). However, when patients were divided into remission and grumbling (active) groups, much higher GPR97 levels were detected in N $\varphi s$ of patients with signs of active disease (Fig. 1f). Moreover, GPR97 expression levels also correlated positively to the ANCA titers of patients (Fig. 1g). Altogether, upregulated GPR97 expression in activated $\mathrm{N} \varphi s$ is positively associated with disease activity of various inflammatory disorders.

Neutrophils are activated by GPR97 binding to a putative ligand expressed exclusively in a human neutrophil subpopulation 
To search for the potential GPR97-specific ligands, we screened a panel of cell lines and primary cells in a fluorescence-activated cell sorting (FACS)-based ligand-binding assay $^{19}$. A chimeric GPR97 ${ }^{\mathrm{E}}-\mathrm{mFc}$ protein containing the human GPR97-ECR fused with a mouse fragment crystallizable (Fc) was used as the probe (Fig. 2a, and Supplementary Fig. 1a). From over 30 different cell samples screened, we successfully identified a putative GPR97-ligand expressed in a unique bimodal pattern exclusively on human $\mathrm{N} \varphi$ surfaces (Fig. 2b, c, and Supplementary Fig. 1b). Intriguingly, the GPR97-ligand ${ }^{+} \mathrm{N} \varphi$ subpopulation ranged from $40 \sim 90 \%$ among different donors but remained rather constant in the same individuals over time (Fig. 2d). Ligand-binding was not detected in human $\mathrm{N} \varphi$ s probed by mouse $\mathrm{Gpr} 97^{\mathrm{E}}-\mathrm{mFc}$, nor in mouse $\mathrm{N} \varphi s$ probed by human and mouse GPR97 ${ }^{\mathrm{E}}-\mathrm{mFc}$ probes (Supplementary Fig. 1c). Hence, the interaction of GPR97 and its putative ligand is a human $\mathrm{N} \varphi$-specific feature and the expression of GPR97ligand is strictly regulated in $\mathrm{N} \varphi s$.

Importantly, incubation of resting $\mathrm{N} \varphi s$ with $\mathrm{GPR} 97^{\mathrm{E}}-\mathrm{mFc}$ triggered robust activation, resulting in several distinct phenotypic manifestations. These include cell shape changes, enhanced production of reactive oxygen species (ROS) and IL-8, increased myeloperoxidase (MPO) activity, as well as expressional changes of multiple $\mathrm{N} \varphi$ activation markers such as CD62L, Mac-1, CD54, and CXCR1 (Fig. 2e-j, and Supplementary Fig. 1d-g). Notably, IL-8 production was up-regulated comparably by $\mathrm{N} \varphi s$ treated with soluble or immobilized GPR97 ${ }^{\mathrm{E}}-\mathrm{mFc}$ in a dose- and time-dependent manner (Fig. 2j, and Supplementary Fig. 1h). In summary, a putative GPR97-specific ligand is identified exclusively in a distinct human $N \varphi$ subset whose interaction with $\mathrm{GPR}^{\mathrm{E}} \mathrm{-mFc}$ induces inflammatory activation.

\section{GPR97 is a specific binding partner and allosteric activator of neutrophil mPR3}

The known ligands of aGPCRs include lipids, glycosaminoglycans, and proteins ${ }^{15}$. To delineate the putative GPR97-ligand, far-western blot analysis of $\mathrm{N} \varphi$ proteins was performed using GPR97 ${ }^{\mathrm{E}}-\mathrm{mFc}$ as a probe. Three distinct signals were detected specifically in the membrane fraction, including a prominent $29 \mathrm{kDa}$ protein and two weaker signals of $\sim 52$ and $\sim 140$ kDa proteins (Fig. 3a), suggesting that the GPR97-ligand is likely a membrane protein(s). To date, CD177 ( 50-60 kDa), PR3 ( $29 \mathrm{kDa})$, and olfactomedin 4 (OLFM4)( $57 \mathrm{kDa})$ are all known to show a bimodal profile in human $\mathrm{N} \varphi s$ hence are strong GPR97-ligand candidates $^{20}$. The GPI-linked CD177 is a $\mathrm{N} \varphi$ restricted Ly6 receptor family member expressed in a characteristic $0 \sim 100 \%$ pattern in 
different individuals due to novel genetic mechanisms ${ }^{21,22}$. The intracellular elastaselike PR3 is somewhat exocytosed and forms mPR3 on resting $N \varphi s$ mainly by binding to CD177, thus displaying a CD177-like expression pattern ${ }^{6}$. By contrast, OLFM4 is a specific granule resident protein which is secreted upon $\mathrm{N} \varphi$ activation and is not known to tether on the $\mathrm{N} \varphi$ membrane $\mathrm{e}^{23}$.

Interestingly, the putative GPR97-ligand was sensitive to phosphoinositide phospholipase C (PI-PLC) digestion that cleaves the GPI-linker (Fig. 3b, and Supplementary Fig. 2a). Moreover, the GPR97-ligand was detected exclusively in the $\mathrm{CD}_{177^{+}}$and $\mathrm{mPR}^{+} \mathrm{N} \varphi$ subsets, showing closely matched expression profiles and strong co-localization with CD177 and mPR3 on N $\varphi$ surfaces (Fig. 3c, and Supplementary Fig. 2b). By contrast, only $20 \sim 40 \%$ of $N \varphi s$ expressing OLFM4 or GPI-linked CD55 were GPR97-ligand $^{+}$(Supplementary Fig. 2c). Finally, morphological changes and IL-8 upregulation induced by GPR97 ${ }^{\mathrm{E}}-\mathrm{mFc}$ were detected solely in the $\mathrm{CD} 177^{+}$but not $\mathrm{CD}^{-177^{-}}$ $\mathrm{N} \varphi$ subset (Fig. $3 \mathrm{~d}$, e, and Supplementary Fig. $2 \mathrm{~d}$ ). These results suggested strongly a direct interaction of GPR97 ${ }^{\mathrm{E}}-\mathrm{mFc}$ with either CD177 or mPR3. Thereafter, far-western blot analyses revealed specific binding of GPR97 ${ }^{\mathrm{E}}-\mathrm{mFc}$ to PR3 but not CD177 (Fig. 3f). We subsequently verified the specific GPR97-PR3 interaction using two different PR3binding assays, namely the FACS-based ligand-binding assay performed in CD177- and GPR97-expressing HEK-293T cells and the ELISA-like protein-protein binding assay (Fig. $3 g-i$, and Supplementary Fig. 2e, f). Both assays showed that GPR97, like CD177, binds to PR3 directly and specifically, albeit much less efficiently. Altogether, we conclude that GPR97-ECR interacts specifically with the PR3 moiety of the mPR3 (PR3-CD177) complex on human $\mathrm{N} \varphi$ surfaces.

The enzymatic activity of $\mathrm{mPR} 3$ is much reduced compared to soluble PR3 due to the PR3-CD177 interaction ${ }^{24}$. This prompted us to investigate the potential role of mPR3 activity in GPR97-induced N $\varphi$ activation and the possible effect of GPR97-mPR3 binding to its proteolytic activity. Surprisingly, GPR97-elicited N $\varphi$ activation was significantly attenuated in the presence of broad-spectrum serine protease inhibitors including $\alpha 1$ antitrypsin (A1AT), aprotinin, and TPCK, but not the cysteine protease inhibitor E-64 (Fig. 3j, and Supplementary Fig. 3a). Accordingly, two endogenous NSP inhibitors, elafin and secretory leukocyte peptidase inhibitor (SLPI), were employed next. SLPI only inhibits NE and CG, while elafin inhibits NE, CG, and PR3 ${ }^{25}$. Interestingly, IL-8 upregulation was dramatically inhibited in elafin- but not SLPI-treated N $\varphi s$ (Fig. 3k). These findings established that mPR3 protease activity is essential for GPR97-induced $N \varphi$ 
activation and implied that GPR97 binding to mPR3 likely promotes its proteolytic activity. We therefore determined the ex vivo enzymatic activity of mPR3 using a PR3specific FRET-based substrate ${ }^{26}$. As expected, incubation of $\mathrm{N} \varphi s$ with GPR97 ${ }^{\mathrm{E}}-\mathrm{mFc}$ resulted in augmented $\mathrm{mPR} 3$ activities which were inhibited by A1AT and elafin, but not SLPI without apparent degranulation of azurophilic granules (Fig. 3l, m, and Supplementary Fig. 3b-d). Thus, we conclude that GPR97 is a binding partner and allosteric activator of mPR3.

\section{Atomic resolution of the GPR97 extracellular region and mapping of the MPR3- binding domains}

The ECR of most aGPCRs contains an extended N-terminal segment followed by a GAIN domain both of which are able to interact with distinct ligands ${ }^{15}$. Previous structural studies have shown that the canonical GAIN domain is evolutionarily conserved and includes $A$ and $B$ subdomains ${ }^{27}$. Subdomain $A$ typically consists of $6 \alpha$-helices, while subdomain $B$ comprises a twisted $\beta$-sandwich made of $13 \beta$-strands and 2 small $\alpha$ helices ${ }^{27}$. Nevertheless, structural analyses of GPR56/ADGRG1 and GPR126/ADGRG6 have revealed a smaller subdomain $A$ with fewer $\alpha$-helices ${ }^{28,}{ }^{29}$. Interestingly, our bioinformatic analyses predicted that GPR97 contains an even smaller GAIN domain than GPR56 and GPR126.

To gain a better insight into the structural organization and mPR3-binding characteristics of GPR97-ECR, we determined its atomic structure (residues 1-264) at 3.37 Å resolution using X-ray crystallography (Fig. 4a, b, and Supplementary Fig. 4a-d). The structural analyses showed the GPR97-GAIN domain retained a complete subdomain $B$ but contained only one $\alpha$-helix in subdomain $A$, approximately where $\alpha 6$ of the canonical GAIN-subdomain A would be located (Supplementary Fig. 4e and 5a). The electron density map also confirmed that GPR97-ECR is partially auto-proteolysed as indicated by previous results (Fig. 4c, and Supplementary Fig. $1 \mathrm{a}$ and $4 \mathrm{f})^{18}$. Clear electron density was visible above the $\alpha$ helix of the subdomain $A$ (Fig. $4 d$ ), but not where $\alpha 4-\alpha 5$ helices of a canonical subdomain $A$ would lie, suggesting the presence of a small N-terminal domain (NTD) on top of the GAIN domain. The NTD contains four cysteine residues (C32, C50, C54, and C62) that are highly conserved among GPR97 orthologs (Supplementary Fig. 5b). To aid the model solution of this domain, SulphurSAD phasing was used to precisely locate the cysteine residues. The resulting model shows that the single helix of the subdomain $A$ (helix $\alpha 6$ in canonical GAIN domains) 
packs against subdomain $B$ and acts as an anchor for the upstream helix of the NTD. A cysteine bridge stabilises the packing of the NTD helix against the subdomain A helix (C54-C62) (Fig. 4a, b). The NTD helix is further stabilised by extensive hydrophobic interactions with the GAIN domain, involving the residues Y39, L44, F51 from the NTD and L67, Y70, W71, Y74, L78 and F91 from the GAIN domain, and a cysteine bridge between the helix and the N-terminal loop of the NTD (C32-C50). These hydrophobic residues are highly conserved specifically among GPR97 orthologs (Supplementary Fig. $4 e$ and $5 b$ ). Structural alignment of GPR97-ECR with other GAIN domains solved to date suggests that the $\alpha$-helix of NTD differs in the orientation of theirs (Fig. 4e, and Supplementary Fig. $4 \mathrm{~g}$ and $4 \mathrm{~h})^{27,28,29}$. Taken together, the GPR97-ECR consists of a small cysteine-stabilised helical NTD followed by an unusually small GAIN domain that consists of a subdomain A with one $\alpha$-helix and a typical subdomain B with $13 \beta$ strands (Fig. 4a and 4f).

To map the mPR3-binding region(s), we generated domain-swapped mFc-fusion probes by interchanging the GAIN domains of GPR97 and GPR56, two closely related ADGRG subfamily members (Fig. 4g, and Supplementary Fig. 6a). A similar ligand-binding signal was detected by the GPR97 ${ }^{\text {NTD }} /$ GPR56 $6^{\text {GAIN }}-\mathrm{mFc}$ and GPR56 $6^{\text {PLL } / G P R 97 ~}{ }^{\text {GAIN }}-\mathrm{mFc}$ probes as did GPR97 $-\mathrm{mFc}$, while GPR56 $-\mathrm{mFc}$ showed nil binding (Fig. 4g). These results suggested that both the GPR97-NTD and -GAIN domains contain one independent mPR3-binding region, respectively. Domain-truncated GPR97-mFc probes were used subsequently to confirm the two different domains can indeed bind to MPR3 individually (Fig. 4h, and Supplementary Fig. 6a). Unexpectedly however, these domain-swapped and -truncated mFc-fusion proteins induced much weaker $N \varphi$ activation phenotypes compared to the full-length GPR97 ${ }^{\mathrm{E}}-\mathrm{mFc}$ (Fig. 4i, j, and

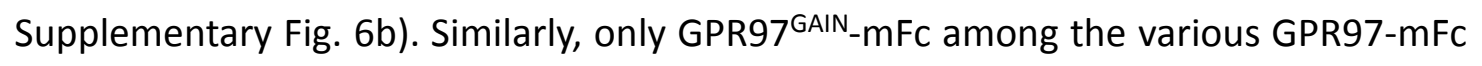
probes stimulated a moderately-increased mPR3 activity (Fig. 4k). We conclude that the maximal GPR97-elicited $\mathrm{N} \varphi$ activation is achieved when mPR3 is bound simultaneously by the two ligand-binding regions in the GPR97-NTD and -GAIN domains.

GPR97-mPR3 interaction requires a macromolecular CD177/GPR97/PAR2/CD16b receptor interactome and triggers PAR2 transactivation in $\mathrm{N} \varphi \mathrm{s}$

As CD177 is incapable of direct signaling due to the lack of a TM moiety, the results above implicated strongly the involvement of an undefined signaling molecule 
triggered by the GPR97-augmented mPR3 in inducing $N \varphi$ activation. In order to identify this signal transducer, a heterologous HEK-293T cell transient expression system was established. Unexpectedly, while exogenous PR3 bound readily to CD177-expressing HEK-293T cells hence forming mPR3, no GPR97 ${ }^{\mathrm{E}}-\mathrm{mFc}$ binding was ever detected in these cells (Fig. 5a). We thus speculated that additional co-receptor(s) expressed in N $\varphi s$ but not HEK293T cells is needed for efficient GPR97-mPR3 binding and likely represents the signaling molecule of interest.

To date, several interacting proteins/substrates of CD177 and PR3, including the endothelial protein C receptor (EPCR), CD16b (FcyRIIIb), Mac-1 (CD11b/CD18), and PAR2 have been identified in $\mathrm{N} \varphi s^{30,31,32,33}$. Hence, we co-expressed CD177 and these proteins in different combinations in HEK-293T cells. Surprisingly, a positive GPR97mPR3 binding signal was only detected in cells co-expressing the specific combination of CD177, GPR97, PAR2 and CD16b (Fig. 5a, b). Moreover, the mPR3-binding signal was lost when any member of the four-receptor complex was replaced with a different protein of the same receptor types, indicating that every co-receptor is indispensable for efficient GPR97-mPR3 binding (Fig. 5c). Next, the proximity-ligation assay (PLA) was carried out to verify the close association of various receptor-pairs of the CD177/GPR97/PAR2/CD16b complex on the $N \varphi$ membrane (Fig. $5 d$, and Supplementary Fig. 3e). These results indicate that a clustered CD177/GPR97/PAR2/CD16b receptor interactome is minimally required for efficient GPR97-mPR3 binding.

PAR2 is the predominant PAR expressed by human N $\varphi s$ and its activation is known to induce activation phenotypes similar to those triggered by GPR97 ${ }^{\mathrm{E}}-\mathrm{mFc} \mathrm{c}^{12}$. PAR2 hence seemed the most likely signaling transducer activated by GPR97-augmented mPR3. Indeed, the surface PAR2 levels of both N $\varphi s$ and PR3-bound HEK-293T transfectants were significantly reduced when incubated with GPR97 ${ }^{\mathrm{E}}$-mFc (Fig. 5e, f). Most critically, enhanced IL-8 production by GPR97 ${ }^{\mathrm{E}}-\mathrm{mFc}$-treated $\mathrm{N} \varphi s$ was significantly and specifically attenuated in the presence of a functional-blocking anti-PAR2 mAb and PAR2-specific antagonists, while the PAR1 antagonist had no inhibitory effect (Fig. 5g). These results clearly demonstrated that PAR2 is not only the essential component of the CD177associated interactome, but also is the signaling receptor targeted by the GPR97augmented mPR3. Thus, we propose a novel GPR97-PAR2 transactivation mechanism that involves PR3 and the CD177/GPR97/PAR2/CD16b interactome. More precisely, GPR97 binds specifically to mPR3 presented by the CD177-associated receptor 
interactome and promotes its enzymatic activity which in turn cleaves and activates PAR2 (Fig. 5h).

\section{Up-regulated GPR97 and PAR2 expression induces GPR97-PAR2 transactivation in activated $\mathrm{N} \varphi \mathrm{s}$}

Resting human $\mathrm{N} \varphi s$ are generally inert suggesting no competent induction of GPR97PAR2 transactivation which is almost certainly regulated by the expression levels of individual members of the CD177-associated receptor interactome. Indeed, our results showed that while CD16b, mPR3, and CD177 were highly expressed in all or some (for mPR3 and CD177) resting NQS, GPR97 and PAR2 were weakly, sometimes barely, expressed (Fig. 6a, and Supplementary Fig. 7a). The low expression levels of GPR97 and PAR2 in resting N $\varphi$ s likely limited the efficient induction of GPR97-PAR2 transactivation. In line with previous results, GPR97 and PAR2 expression was up-regulated significantly when resting $\mathrm{N} \varphi s$ were stimulated by degranulation stimulants of azurophilic granules or by heat-aggregated IgGs (algGs) via an Fc receptor (FcR)-dependent mechanism (Fig. $6 a, b)$. Consequently, the PLA analyses detected significantly increased interaction signals of specific receptor-pairs of the CD177-associated interactome in algG-activated $\mathrm{N} \varphi s$ in comparison to resting N $\varphi s$ (Fig. 6c). Importantly, the up-regulated IL-8 production induced by algG-stimulated N $\mathrm{ps}$ was attenuated in the presence of the FcR blocker, serine protease inhibitors, PAR2 antagonists, as well as functional blocking anti-PAR2 and anti-GPR97 mAbs, suggesting the efficient induction of GPR97-PAR2 transactivation (Fig. 6d).

To evaluate the potential clinical relevance of the FcR-induced GPR97-PAR2 transactivation, N $\varphi s$ were incubated with purified PR3-ANCA IgGs which were known

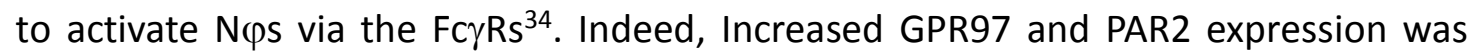
detected in resting N $\varphi$ s incubated with PR3-ANCA, but not MPO-ANCA IgGs, in a FcRdependent manner (Supplementary Fig. 7b, c). Critically, PR3-ANCA IgGs caused extensive $N \varphi$ aggregates and produced significantly more IL- 8 which was attenuated in the presence of FcR blocker, serine protease inhibitors, or functional blocking anti-PAR2 and anti-GPR97 mAbs (Fig. 6e, f, and Supplementary Fig. 7d and 7e). Altogether, we conclude that PAR2 and GPR97 were stored mostly in the azurophilic granules and were translocated to cell surfaces when $\mathrm{N} \varphi \mathrm{s}$ received specific activation signals. The increased PAR2 and GPR97 expression subsequently promoted clustering of the CD177- 
associated receptor interactome to induce GPR97-PAR2 transactivation, leading to further $\mathrm{N} \varphi$ activation.

\section{GPR97-PAR2 transactivation promotes $\mathrm{N} \varphi$-mediated anti-microbial activity and endothelial cell activation and dysfunction}

To investigate the potential innate immune function of GPR97-PAR2 transactivation, the effects of GPR97 ${ }^{\mathrm{E}}-\mathrm{mFc}$ on the bacterial uptake and killing abilities of $\mathrm{N} \varphi s$ were examined. As shown, the engulfment of several bacteria species including $E$. coli, $S$. typhimurium, and S. aureus was greatly enhanced in $\mathrm{N} \varphi$ s incubated with GPR97 ${ }^{\mathrm{E}}-\mathrm{mFc}$, but not the mFc control (Fig. 7a). Similarly, the bacterial killing assay showed that GPR97 $^{\mathrm{E}}-\mathrm{mFc}$ treatment significantly facilitates the elimination of S. typhimurium and $S$. aureus by NQs (Fig. 7b).

Uncontrolled and excess activated $\mathrm{N} \varphi s$ were frequently associated with adverse tissue damage seen in various inflammatory disorders ${ }^{35}$. To delineate the effect of GPR97PAR2 transactivation on the vasculatures, the activation and dysfunction of endothelial cells were investigated using the HUVEC-N $\varphi$ co-culture system. HUVECs were significantly activated when co-cultured with $\mathrm{N} \varphi \mathrm{s}$ in the presence of GPR97 ${ }^{\mathrm{E}}-\mathrm{mFc}$ (Fig. 7c). Moreover, GPR97 ${ }^{\mathrm{E}}-\mathrm{mFc}$ treatment greatly promoted endothelial permeability and dysfunction (Fig. 7d). Finally, similar increased endothelial permeability was identified in HUVEC-N $\varphi$ co-culture in the presence of algGs which was dramatically attenuated in the presence of FcR blocker, PAR2 antagonists, or functional blocking anti-PAR2 and anti-GPR97 mAbs (Fig. 7e). In summary, we conclude that the mPR3-mediated GPR97PAR2 transactivation in N $\varphi$ s plays a role in the anti-microbial functions as well as endothelial cell activation and dysfunction.

\section{DISCUSSION}

In this study, GPR97 was deorphanized as the binding partner and allosteric activator of mPR3 which in turn activated PAR2 on Nps (Fig. 2, 3). This novel mPR3-mediated GPR97-PAR2 transactivation adds a significant component to the repertoire of $N \varphi$ activation mechanisms (Fig. 5-7).

PAR2 activation is normally elicited by proteolytic enzymes including soluble proteases such as trypsin and microbial proteases as well as single-TM or GPI-linked membrane sheddases such as matriptase and testisin ${ }^{36,37}$. The active coagulation protein complex 
containing tissue factor (TF)/factor VIla (FVIla)/factor Xa (FXa) could also act as a potent PAR2 activator ${ }^{38}$. In this case, PAR2 is transactivated indirectly by the TF/FVIIa/Xa complex via its proteolytic activation of the matriptase zymogen ${ }^{39}$. By contrast, PAR1-PAR2 transactivation was achieved via the interaction of PAR2 with the thrombin-exposed tethered ligand of PAR1 ${ }^{14}$. Interestingly, the GPR97-PAR2 transactivation process also involves a unique protein complex, here containing a serine protease, two GPI-linked receptors, and an aGPCR (Fig. 5h). PAR2 is expressed ubiquitously and its activation has been shown to play multiple immune-regulatory roles including $\mathrm{N} \varphi$ activation and inflammatory cytokine production $40,41,42$. Critically, $\mathrm{N} \varphi$ activation via the TF/FVIIa/PAR2 axis resulted in trophoblast injury and fetal death in an APS animal model ${ }^{11}$. Our findings of the up-regulation of GPR97 and PAR2 expression in activated N $\varphi s$ suggested that the GPR97-PAR2 transactivation mode is likely a common $\mathrm{N} \varphi$ activation mechanism associated with inflammatory diseases (Fig. $1,6)$.

The absolute requirement of the clustered PR3/CD177/GPR97/PAR2/CD16b interactome for efficient GPR97-PAR2 transactivation is surprising (Fig. 5), but it is not without precedent. In fact, it is highly analogous to the selective involvement of the GPI-anchored RECK and GPR124/ADGRA2 receptor complex in mediating the Frizzled 4 (FZD4)/Low-density lipoprotein receptor-related protein 5 (LRP5)-dependent Wnt7aspecific bioactivity for CNS angiogenesis and blood-brain barrier integrity ${ }^{43,44}$. Coincidentally, the two aGPCR-GPCR protein complexes all consist of one soluble protein (PR3/Wnt7) and 4 receptor molecules including one GPI-linked receptor (CD177/RECK), one aGPCR (GPR97/GPR124), and one GPCR (PAR2/FZD4).

GPCR transactivation is an efficient means to expand the signaling outputs and usually involves the activation of another receptor type such as receptor tyrosine kinases (RTKs) by GPCRs ${ }^{45}$. Nevertheless, recent studies have shown that transactivation of GPCRs can also be induced by RTKs and other GPCRs ${ }^{46,47}$. GPCR transactivation of RTKs typically involves the activation of extracellular metalloproteases, which cleave and release membrane-bound protein ligands of $\mathrm{RTKS}^{48}$. In line with this, it is intriguing to note the involvement of GPR97-augmented mPR3 enzymatic activity in the PAR2 transactivation process (Fig. 3). Interestingly, approximate analogies were also noted in the Wnt7a-RECK-GPR124 complex in which RECK acted as a competitive inhibitor of Wnt7a signaling by specifically ligating and segregating Wnt7a from FZD4. GPR124 engagement efficiently realigned the RECK-Wnt7a complex to be available for FZD4 
recognition hence permitting signaling ${ }^{44}$. With these in mind, we suggest that the aGPCR-GPCR transactivation mechanism symbolizes a previously unappreciated GPCR activation paradigm beyond the classical ligand-induced receptor activation.

GPR97 belongs to the ADGRG subgroup of aGPCR family which contains some of the smaller-sized members including GPR56 and GPR114 ${ }^{49}$. Although the GAIN domain has been identified as an evolutionarily conserved structural fold sufficient for GPS autoproteolysis, the minimal domain structure needed for the unusual auto-proteolytic reaction remained to be fully elucidated ${ }^{27}$. Our analyses of GPR97-ECR showed that a well-conserved subdomain B plus a small one $\alpha$-helix-containing subdomain A are competent for efficient GPS auto-proteolysis (Fig. 4). Recently, the cryo-electron microscopy structures of glucocorticoid-bound GPR97-Go complexes have revealed the first 3D structure of an aGPCR-7TM region ${ }^{50}$. With the structural resolution of GPR97-ECR reported here, it is possible now to build a complete structural feature of a full-length aGPCR.

PR3 is the most abundant protease of azurophilic granules with versatile intracellular and extracellular functions ${ }^{4}$. Specific binding of mPR3 by PR3-ANCA which ligated and activated FcyRs was considered as the major disease mechanism of $\mathrm{N} \varphi$ activation in $\mathrm{GPA}^{34}$. Despite these, little is known of the physiological function of mPR3. Our findings of the tunable enzymatic activity of mPR3 by GPR97 and the subsequent PAR2 transactivation hence uncover a novel role for mPR3 in immune regulation (Fig. 3-7). Paradoxically, the outcomes of PR3-mediated PAR2 cleavage seemed to depend critically on the cell types studied. Indeed, PR3 digestion was shown to disarm PAR2 for subsequent trypsin-mediated activation in kidney epithelial cells ${ }^{51}$. On the other hand, PAR2 was efficiently activated by PR3 in immature dendritic cells (iDCs) ${ }^{40}$ and by the IL-32 $\gamma /$ PR3 complex in THP-1 cells ${ }^{52}$. In the future, it could be a valuable advance to reveal the mechanistic detail of GPR97-PAR2 transactivation and to survey other potential protein substrates of the GPR97-augmented mPR3 activity.

In view of the unique human $\mathrm{N} \varphi$-specific feature of GPR97-mPR3 interaction (Fig. 2, and Supplementary Fig.1c), it was surprising to find novel interspecies differences in several members of the PR3/CD177/GPR97/PAR2/CD16b interactome. Human PR3 differed from gibbon and murine PR3s by containing a distinctive hydrophobic patch which mediated its interaction with $\mathrm{CD} 177^{53}$. Unlike the $\mathrm{N} \varphi$-restricted expression of GPR97 in human, Adgrg3/Gpr97 was involved in B-lymphocyte fate decision ${ }^{54}$, obesityassociated macrophage inflammation ${ }^{55}$, and lymphatic endothelial cell migration in 
mice ${ }^{56}$. Finally, CD16b is the $\mathrm{N} \varphi$-specific GPI-anchored Fc $\gamma R$ III expressed only in human and not mouse ${ }^{57}$. These unique features point to the singularity of the GPR97-PAR2 transactivation mechanism in regulating human $N \varphi$ activation. Moreover, as proteolytic enzymes and GPCRs belong to two major families of molecular drug targets, the PR3/CD177/GPR97/PAR2/CD16b receptor interactome represents a vital multitarget complex for the development of potential therapeutics to modulate human $\mathrm{N} \varphi$ mediated inflammatory diseases ${ }^{58,59}$.

\section{METHODS}

\section{Reagents and antibodies}

All chemicals and reagents were purchased from Sigma (St. Louis, MO, USA) and Invitrogen (CA, USA) unless specified otherwise. E-64 (\#78434) was from Thermo Fisher Scientific. Human proteinase 3 (PR3) (\#16-14-161820) was purchased from Athens Research \& Technology, Inc. (Athens, Georgia, USA). Human Fc receptor blocker (Human BD Fc Block ${ }^{\mathrm{TM}}$, \#564220) was from BD Biosciences (New Jersey, USA). Antibodies used in this study were listed in the supplementary Table 1.

\section{Cell culture}

All cell culture media and supplements, including fetal calf serum (FCS), L-glutamine, penicillin and streptomycin were purchased from Invitrogen. Cell lines used in this study (listed in the supplementary Table 2) were purchased from the American Type Culture Collection (Manassas, VA, USA) or Bioresource Collection and Research Center (Hsinchu, Taiwan) and cultured in conditions as suggested.

\section{Construction of expression vectors and cell transfection}

Mammalian expression constructs encoding the mFc only as well as various GPR97-, GPR56-, and EMR2-mFc fusion proteins were generated in the pcDNA3.1-mFc vector using standard molecular biology technologies as described previously ${ }^{19}$. Genespecific oligonucleotide primers used to construct the $\mathrm{mFc}$-fusion protein expression vectors were listed in the supplementary Table 3. For the construction of expression vectors encoding GPR97-TM7-EGFP and GPR97-myc, the full-length human GPR97 cDNA was cloned into the pEGFP-N1 (Clontech) and pcDNA3.1/myc-His A (Invitrogen) vectors, respectively. Expression vectors encoding the PAR2, EPCR, Mac-1 (CD11b/CD18), CD16b, and CD55 receptors were purchased from Sino Biological Inc. 
(Beijing, China). The CD177 and FRP1 expression constructs were gifts from Dr. Ralph Kettritz of Charité of Universitätsmedizin Berlin, Germany and Dr. TL Hwang of Chang Gung University, Taiwan, respectively. The EMR2 expression construct was described previously ${ }^{60}$. For transient cell transfection, HEK-293T cells were transfected with purified plasmid DNAs using Lipofectamine ${ }^{\text {TM }}$ or Lipofectamine ${ }^{\text {TM }} 2000$ (Invitrogen) as described elsewhere.

\section{Generation and purification of the $\mathrm{mFc}$-fusion proteins}

All recombinant $\mathrm{mFc}$-fusion proteins were purified from conditioned media of transiently transfected HEK293-T cells using the Protein A-Sepharose affinity chromatography as described previously ${ }^{19}$. Briefly, HEK-293T cells were transfected with the expression constructs of interest using the calcium phosphate-based transfection method. Following transfection, cells were cultured in serum-free OPTIMEM medium for 4-5 days and conditioned media was collected, centrifuged, and passed through the Protein A-Sepharose affinity column (nProtein A Sepharose ${ }^{\mathrm{TM}} 4$ Fast Flow, GE Healthcare), followed by extensive washes with washing buffer (50 mM Tris- $\mathrm{HCl} \mathrm{pH} \mathrm{7.4,} 10 \mathrm{mM} \mathrm{CaCl}_{2}, 150 \mathrm{mM} \mathrm{NaCl}$ ). The $\mathrm{mFc}$-fusion protein was eluted and subjected to dialysis in the Slide-A-Lyzer ${ }^{\circledR}$ Dialysis Cassettes (Thermo Scientific) in washing buffer for $24 \mathrm{~h}$ at $4^{\circ} \mathrm{C}$, filtered, and stored at $-80^{\circ} \mathrm{C}$ until use.

\section{Purification of blood neutrophils}

Human neutrophils ( $\mathrm{N} \varphi \mathrm{s}$ ) were isolated from fresh venous blood donated by healthy volunteers and diseased patients as indicated. All experimental procedures were approved by the Chang Gung Memorial Hospital Ethics Committee (CGMH IRB No: 201701852BO, 201901358BO, 201901293BO, and 202002255B0) and performed according to the guidelines set by the Committee. In brief, blood samples were drawn into the collection tubes coated with sodium heparin and $\mathrm{N} \varphi$ isolation was performed using Polymorphprep ${ }^{\mathrm{TM}}$ density gradient centrifugation (Axis-Shield, Oslo, Norway) as described previously ${ }^{18}$. For the isolation of murine $\mathrm{N} \varphi s$, blood was collected by cardiac puncture and separated by Ficoll (GE Healthcare) gradient separation. Following the lysis of the remnant erythrocytes, isolated Nps were checked for purity by flow cytometry with specific surface markers and resuspended in RPMI medium containing $10 \%$ fetal bovine serum (FBS) for all following experiments unless otherwise denoted. The isolation procedure routinely produced a $>95 \%$ pure and viable $\mathrm{N} \varphi$ population. 
When indicated, isolated human $\mathrm{N} \varphi s$ were further subjected to the magnetic cell sorting (MACS) separation of $\mathrm{CD} 177^{+}$and $\mathrm{CD} 177^{-}$sub-populations using PE-conjugated anti-CD177 Ab coupled to the MACS MicroBeads and Separator (Miltenyi Biotec, Bergisch Gladbach, Germany) according to the manufacturer's recommendations.

\section{Immunofluorescence assay and immunohistochemistry}

Freshly isolated human $\mathrm{N} \varphi \mathrm{s}\left(1 \times 10^{6}\right.$ cells $\left./ \mathrm{mL}\right)$ were treated as described and then fixed with $4 \%$ paraformaldehyde/PBS at $4^{\circ} \mathrm{C}$ for $20 \mathrm{~min}$. Cells were then incubated with the blocking buffer (1\% BSA, 5\% normal goat serum (NGS) in PBS) for $1 \mathrm{~h}$ at $4^{\circ} \mathrm{C}$, then with indicated primary antibody $(5 \mu \mathrm{g} / \mathrm{mL})$ diluted in blocking buffer for $1 \mathrm{~h}$ at $4{ }^{\circ} \mathrm{C}$. Following extensive washes in cold PBS, cells were incubated for $1 \mathrm{~h}$ at $4{ }^{\circ} \mathrm{C}$ with fluorescence-labeled secondary antibody diluted in blocking buffer at a predetermined optimal concentration. Cells were washed thoroughly before being spun onto slides. Fluorescence images were taken by Fluoview FV10i (Olympus) or the LSM780 confocal microscope system (ZEISS). Immunohistochemistry was carried out on formalin-fixed, paraffin-embedded tissue sections (4 $\mu \mathrm{m})$ using G97-A mAb (5 $\mu \mathrm{g} / \mathrm{mL}$ ) as described previously ${ }^{18}$. All tissue staining procedures were performed in an automated immunostainer (BOND-MAXTM, Leica Biosystem). Immunoreactivity was assessed independently by two expert pathologists. N $\varphi s$ in capillaries were found to display strong GPR97 expression constantly. Therefore, the GPR97 expression level of tissue infiltrating $\mathrm{N} \varphi \mathrm{s}$ (TIN $\varphi s$ ) was scored as follows: 1+, if GPR97 expression was far weaker than that of intravascular N $\varphi ; 2+$, if GPR97 expression was weaker than that of intravascular N $\varphi s ; 3+$, if the GPR97 expression intensity was equal to that of intravascular $\mathrm{N} \varphi$; 4+, if GRP97 expression was stronger than that of intravascular $\mathrm{N} \varphi \mathrm{s}$.

\section{Flow cytometry analysis and the FACS-based ligand-binding assay}

All procedures were performed at $4^{\circ} \mathrm{C}$. Cells were blocked in ice-cold blocking buffer (1\% BSA/5\% NGS in PBS) for $1 \mathrm{~h}$. For the standard flow cytometry analysis, cells were incubated sequentially with the primary $A b$ of interest and appropriate fluorescencelabeled secondary Ab diluted in blocking buffer at pre-determined optimal concentrations for $1 \mathrm{~h}$. For the analysis of GPR97 expression in blood N $\varphi$ s of normal controls and diseased patients, blood leukocytes were obtained following RBC lysis with ACK buffer and washed twice with ice-cold PBS. Cells $\left(1 \times 10^{6} /\right.$ reaction) were stained with a mixture of aqua fluorescent reactive dye (MAN0006891, Life 
Technologies), anti-CD16b-FITC (130-126-529, Miltenyi Biotec), anti-CD66b-PerCPCy5.5 (305108, Biolegend), and anti-GPR97 (G97-A) conjugated with APC (Ab201807, Abcam) for $20 \mathrm{~min}$. Fluorescent minus one controls include GPR97 and aqua fluorescent reactive dye only control were included for background determination. The fluorescence-labeled cells were analyzed by BD FACSCanto II flow cytometry system. For the cellular ligand-binding assay, cells were incubated with the mFc-fusion protein probe $(10 \mu \mathrm{g} / \mathrm{mL})$ as the primary $A b$ in blocking buffer for $2 \mathrm{~h}$. Where indicated, cells were pretreated or treated simultaneously with various reagents in the presence of the probes. Fluorescence-conjugated goat anti-mouse $\operatorname{lgG}(2 \mu \mathrm{g} / \mathrm{mL})$ was used as the $2^{\text {nd }} A b$. Following extensive washes between each incubation step, cells were washed lastly in cold PBS and subjected to analysis by FACScan flow cytometer (BD Biosciences). Positive ligand-binding was determined as the shift of fluorescence signals. The $\mathrm{mFc}$ protein was always included as a negative control.

\section{Phenotypic analyses of neutrophils}

Freshly isolated human $\mathrm{N} \varphi \mathrm{s}\left(1 \times 10^{6}\right.$ cells $\left./ \mathrm{mL}\right)$ were suspended in RPMI complete medium and incubated with soluble GPR97 ${ }^{\mathrm{E}}-\mathrm{mFc}$, control $\mathrm{mFc}$ protein $(10 \mu \mathrm{g} / \mathrm{mL})$, or $\mathrm{f}-\mathrm{MLF}\left(10^{-6} \mathrm{M}\right)$ at $37^{\circ} \mathrm{C}$ for $3 \mathrm{~h}$. For the expressional analysis of CD markers, cells were fixed by $4 \%$ paraformaldehyde/PBS at $4^{\circ} \mathrm{C}$ for $20 \mathrm{~min}$ and stained with fluorescenceconjugated $\mathrm{CD}$ markers as indicated using the standard flow cytometry protocol. For the analysis of $\mathrm{N} \varphi$ morphological changes, live cells were either collected at $1 \mathrm{~h}$ after incubation and photographed under the inverted microscopy, or analyzed directly by FACScan flow cytometer and shown as FSC/SSC. Alternatively, cells were fixed and subjected to the Wright-Giemsa stain, or permeabilized and blocked in the blocking buffer (1\% BSA, 5\% NGS, $0.1 \%$ saponin in PBS) for $30 \mathrm{~min}$, then incubated with Phalloidin-TRITC $(10 \mu \mathrm{g} / \mathrm{mL})$ and analyzed by flow cytometer as well as Fluoview FV10i (Olympus). For IL-8 production, culture supernatant was collected at $3 \mathrm{~h}$ incubation and subjected to the ELISA analysis.

\section{Analyses of reactive oxygen species production and myeloperoxidase activity}

Freshly isolated human $\mathrm{N} \varphi \mathrm{s}\left(2 \times 10^{6}\right.$ cells $\left./ \mathrm{mL}\right)$ were resuspended in PBS supplemented with $0.2 \%$ BSA and $5 \mathrm{mM}$ glucose, and incubated with $2 \mu \mathrm{M}$ dihydrorhodamine-123 (DHR123; Molecular Probes \#D23806) for $30 \mathrm{~min}$ at RT. Cells were then incubated respectively with $\mathrm{GPR}^{\mathrm{E}} \mathrm{-mFc}$ or control $\mathrm{mFc}$-fusion protein $(10 \mu \mathrm{g} / \mathrm{mL})$ for $30 \mathrm{~min}$ at 
$37^{\circ} \mathrm{C}$. Cells were then stimulated with or without fMLF $\left(10^{-6} \mathrm{M}\right)$ for $15 \mathrm{~min}$ before being placed on ice to stop the reaction. The accumulation of reactive oxygen species (ROS) represented by fluorescent oxidized DHR123 was immediately measured by flow cytometry as described previously ${ }^{18}$.

The myeloperoxidase (MPO) enzyme activity was analyzed using the colorimetric MPO activity assay kit (BioVision) performed exactly as suggested by the manufacturer. Briefly, freshly isolated human $\mathrm{N} \varphi s\left(2.5 \times 10^{6}\right.$ cells $\left./ \mathrm{mL}\right)$ were resuspended in PBS supplemented with $0.2 \% \mathrm{BSA}$ and $5 \mathrm{mM}$ glucose. Cells were then incubated with $\mathrm{GPR}^{\mathrm{E}}-\mathrm{mFc}$ or control $\mathrm{mFc}$ fusion protein $(10 \mu \mathrm{g} / \mathrm{mL})$ for $30 \mathrm{~min}$ at $37^{\circ} \mathrm{C}$. When necessary, cells were first incubated with GPR97 ${ }^{\mathrm{E}}-\mathrm{mFc}$, followed by stimulation with or without fMLF $\left(10^{-6} \mathrm{M}\right)$ for $15 \mathrm{~min}$. Cells were lysed with the MPO assay buffer and then centrifuged at $10,000 \mathrm{~g}$ for $10 \mathrm{~min}$ at $4{ }^{\circ} \mathrm{C}$. Lysate supernatant (10 $\left.\mu \mathrm{L} / \mathrm{sample}\right)$ was added to $40 \mu \mathrm{l}$ of MPO assay buffer in a 96-well plate. The reaction was initiated by the addition of $10 \mu \mathrm{L}$ MPO substrate solution to each well and incubated for $60 \mathrm{~min}$ at RT. Finally, $2 \mu \mathrm{L}$ of stop solution was added, followed immediately with $50 \mu \mathrm{L}$ of Ellman's reagent (5,5'-dithiobis-(2-nitrobenzoic acid), DTNB) for the colorimetric analysis of the MPO activity at $412 \mathrm{~nm}$.

\section{Measurement of IL- 8 by sandwich ELISA}

Freshly isolated human $\mathrm{N} \varphi s\left(1 \times 10^{6}\right.$ cells $\left./ \mathrm{mL}\right)$ were cultured in $\mathrm{RPMI} / 10 \% \mathrm{FBS}$ in the presence of $\mathrm{fMLF}\left(10^{-6} \mathrm{M}\right), \mathrm{mFc}$ or GPR97 ${ }^{\mathrm{E}}-\mathrm{mFc}(10 \mu \mathrm{g} / \mathrm{mL})$ for $3 \mathrm{~h}$ at $37^{\circ} \mathrm{C}$ incubator. When indicated, culture plates were coated with the $\mathrm{mFc}$ fusion proteins at $4{ }^{\circ} \mathrm{C}$ overnight before incubation with $\mathrm{N} \varphi \mathrm{s}$. When necessary, $\mathrm{N} \varphi s$ were pretreated with various reagents/inhibitors such as $\alpha 1$-antitrypsin $(5 \mathrm{mg} / \mathrm{mL})$, aprotinin $(50 \mu \mathrm{g} / \mathrm{mL}), \mathrm{E}-$ $64(50 \mu \mathrm{g} / \mathrm{mL})$ and N-p-Tosyl-L-phenylalanine chloromethyl ketone (TPCK, $10 \mu \mathrm{M})$ for $1 \mathrm{~h}$ at $37^{\circ} \mathrm{C}$ incubator or incubated with elafin ( $\left.4 \mu \mathrm{M}\right)$ and SLPI ( $\left.4 \mu \mathrm{M}\right)$ simultaneously. Cell-free supernatants were collected to determine the concentration of interleukin- 8 (IL-8) by using the sandwich enzyme-linked immunosorbent assay (ELISA) kit (DuoSet ${ }^{\circledR}$ ELISA, R\&D Systems, DY208) according to the protocols suggested by the manufacturer.

\section{Structural analysis of GPR97-ECR}

For crystallisation experiments, human GPR97 ECR (UniProt Q8R0T6, residues 1 to 264) was cloned into the EcoRI-Kpnl cloning sites of vectors from the pHLsec family with its native secretion signal sequence and a C-terminal His6 tag. The cDNA encoding GPR97 
possesses a SNP at residue $447(\mathrm{M} \rightarrow \mathrm{V})$. GPR97 ${ }^{\mathrm{ECR}}$ was expressed in a secreted form in GInTI-deficient HEK293S cells using previously described protocols ${ }^{61}$. Briefly, plasmid DNAs were transfected with PEI in a 1:2 ratio into $3 \mathrm{~L}$ of $90-100 \%$ confluent HEK293S cells. After 10 days, the cell culture medium containing the secreted GPR97 ${ }^{\text {ECR }}$ was clarified by centrifugation and filtration prior to diafiltration into PBS, $20 \mathrm{mM}$ Tris $\mathrm{pH}$ 7.5 and $150 \mathrm{mM} \mathrm{NaCl}$. GPR97 ${ }^{\text {ECR }}$ was then purified by Ni-NTA affinity (HiTrapTM HP, GE Healthcare) and size-exclusion chromatography (SEC) (Superdex 200 16/600, GE Healthcare) in $20 \mathrm{mM}$ Tris $\mathrm{pH} 7.5$ and $300 \mathrm{mM} \mathrm{NaCl}$. The protein purity was assessed by sodium dodecyl sulphate-polyacrylamide gel electrophoresis (SDS-PAGE) and concentrated to $40 \mathrm{mg} / \mathrm{mL}$ using 10,000 MWCO concentrators (Amicon Ultra Centrifugal Filters).

GPR97 $^{\text {ECR }}$ crystals grew in $100 \mathrm{~nL}+100 \mathrm{~nL}$ sitting nanodrops by the vapour diffusion method at $18^{\circ} \mathrm{C}$ in $1.8 \mathrm{M}$ tri-ammonium citrate $\mathrm{pH}$ 7. The crystals were harvested and cryo-protected in $3 \mathrm{M}$ tri-ammonium citrate $\mathrm{pH}$ 7. A native dataset was collected at a wavelength of $0.9763 \AA$ at the European Synchrotron Radiation Facility beamline ID30B. $180^{\circ}$ of data were collected with an exposure of 0.02 seconds per $0.1^{\circ}$ rotation on a PILATUS3 6M (Dectris). The data was processed using DIALS ${ }^{62}$ and AIMLESS ${ }^{63}$. A S-SAD dataset was also collected at a wavelength of $2.75 \AA$ on the long-wavelength beamline $123^{64}$ at Diamond Light Source, UK. $360^{\circ}$ of data were collected with an exposure of $0.1 \mathrm{~s}$ per $0.1^{\circ}$ rotation on a PILATUS $12 \mathrm{M}$ detector (Dectris). The data showed sign of anisotropy and was therefore processed using AUTOPROC ${ }^{65}$ and STARANISO (Tickle, I.J. et al. (2018) STARANISO. Cambridge, United Kingdom: Global Phasing Ltd.) (Supplementary Table 4).

The flexible loops of the GAIN domains of Lphn1, BAI3 and GPR56 (PDB accession numbers 4DLQ, 4DLO, 5KVM respectively) were removed and an ensemble of those trimmed GAIN domains was used as a search model for molecular replacement using the native dataset and PHASER ${ }^{66}$. The solution consisted of two molecules in the asymmetric unit and displayed additional density on top of the GAIN domain, accounting for the small $\mathrm{N}$-terminal domain. To help build this $\mathrm{N}$-terminal domain, molecular replacement using the S-SAD dataset and PHASER ${ }^{66}$ was carried out with the partial model consisting of the GPR97 GAIN domain. The resulting solution was subjected to one round of refinement in autoBUSTER (Bricogne G., et al (2017) BUSTER version 2.10.3. Cambridge, United Kingdom: Global Phasing Ltd. ${ }^{67}$. Electron density maps were improved in PARROT $^{68}$ revealing the missing $\mathrm{N}$-terminal domain. 
Anomalous difference maps calculated with $\mathrm{ANODE}^{69}$ were used to locate sulphur atoms and help manual building in $\mathrm{COOT}^{70}$. All-atom refinement with autoBUSTER (Bricogne G., et al (2017) BUSTER version 2.10.3. Cambridge, United Kingdom: Global Phasing Ltd. $)^{67}$ resulted in the final model containing residues 28-260.

\section{Western and far-western immunoblotting analysis}

Cells were lysed in RIPA lysis buffer (20 mM Tris-HCl pH7.4, $5 \mathrm{mM} \mathrm{MgCl}, 100 \mathrm{mM} \mathrm{NaCl}$, 0.5\% Nonidet P-40, and $1 \mathrm{X}$ Complete Protease Inhibitors) supplemented with $1 \mathrm{mM}$ sodium orthovanadate, $1 \mathrm{mM}$ AEBSF, and $5 \mathrm{mM}$ Levamisole. Lysate proteins were quantified using the Bicinchoninic acid (BCA) protein assay kit (Pierce). Protein samples were separated in $10 \%$ SDS or native PAGE gels and transferred to PVDF membranes. Membranes were then soaked in blocking buffer (PBS containing 5\% nonfat skimmed milk and $0.1 \%$ Tween 20 ) for $1 \mathrm{~h}$ at room temperature. Next, the membranes were incubated with indicated primary Ab or GPR97 ${ }^{\mathrm{E}}-\mathrm{mFc}(5 \mu \mathrm{g} / \mathrm{mL})$ diluted in blocking buffer overnight at $4{ }^{\circ} \mathrm{C}$. EMR2 ${ }^{\mathrm{E}}-\mathrm{mFc}$ or $\mathrm{mFc}(5 \mu \mathrm{g} / \mathrm{mL})$ protein was used as negative controls. Following extensive washes in washing buffer (0.1\% Tween 20 in PBS), membranes were incubated with horseradish peroxidase (HRP)-conjugated goat anti-mouse Fc Ab (1:2000) in blocking buffer. Finally, the specific binding of $A b$ and $\mathrm{mFc}$-fusion proteins on membrane blots was revealed with chemiluminescent HRP Substrate (Millipore WBKLS0500).

\section{Protein extraction of the cytoplasmic and membrane fractions}

All protein isolation procedures are performed at $4{ }^{\circ} \mathrm{C}$. Freshly isolated $\mathrm{N} \varphi s\left(2 \times 10^{7}\right.$ cells) were washed three times with cold PBS, re-suspended in RIPA buffer and passed through a 30-gauge needle for 20 times. Cell lysates were then centrifuged at $700 \mathrm{~g}$ for $3 \mathrm{~min}$ at $4{ }^{\circ} \mathrm{C}$, and the supernatant was collected and subjected to ultracentrifugation at $10,000 \mathrm{~g}$ for $40 \mathrm{~min}$ at $4{ }^{\circ} \mathrm{C}$. The resulting supernatant was collected as the cytoplasmic fraction and the pellet was the membrane fraction. All protein samples were stored at $-80^{\circ} \mathrm{C}$ until use.

\section{FACS-based proteinase 3 (PR3)-binding assay}

HEK-293T cells were transfected with various expression constructs as indicated. HEK$293 T$ transfectants were collected, washed, and incubated in blocking buffer (1\% BSA, $5 \% \mathrm{NGS} / \mathrm{PBS}$ ) for $1 \mathrm{~h}$ at $4{ }^{\circ} \mathrm{C}$ before incubating with commercially available purified PR3 
$(5 \mu \mathrm{g} / \mathrm{mL})$ diluted in blocking buffer for $1 \mathrm{~h}$ at $4{ }^{\circ} \mathrm{C}$. Cells were subjected to extensive washes in cold PBS, and then incubated with the anti-PR3 mAb $(5 \mu \mathrm{g} / \mathrm{ml})($ clone MCPR3-2, Thermo Fisher Scientific MA5-11945) or PeliCluster ANCA (5 $\mathrm{g} / \mathrm{ml}$ )(clone CLB-12.8, Sanquin M1574) for $1 \mathrm{~h}$ at $4{ }^{\circ} \mathrm{C}$. Cells were extensively washed and then incubated with fluorescence-labeled secondary antibody for $1 \mathrm{~h}$ at $4{ }^{\circ} \mathrm{C}$. Finally, cells were washed three times in cold PBS and subjected to analysis by FACScan flow cytometer (BD Biosciences). When indicated, HEK-293T cells were first transfected with the GPR97-TM7-EGFP construct. Transfected cells were then subjected to FACS sorting by FACSAria ${ }^{\mathrm{TM}} \|$ (BDbiosciences) to select the top $30 \%$ highest GPR97expressing (GPR97 ${ }^{\text {high }}$ ) and low GPR97-expressing (GPR97 ${ }^{\text {low }}$ ) cells based on the GFP expression levels. The sorted GPR97 high and GPR97 low HEK-293T cells were used to examine the PR3-binding ability as described above. Similar EMR2 $2^{\text {high }}$ and EMR2 $2^{\text {low }}$ HEK-293T transfectants were included as negative controls.

For the analysis of GPR97-mPR3 binding in transfected HEK-293T cells, cells were transfected as described. Half of the transfected cells were checked by the flow cytometry analysis using receptor-specific Abs for the expression of the specific receptors. The other half of the transfected cells were incubated first with purified PR3 $\left(5 \mu \mathrm{g} / \mathrm{mL}\right.$ ) diluted in blocking buffer for $1 \mathrm{~h}$ at $4{ }^{\circ} \mathrm{C}$, washed, and followed by incubation with $\mathrm{mFc}(10 \mu \mathrm{g} / \mathrm{mL})$ or GPR97 ${ }^{\mathrm{E}}-\mathrm{mFc}(10 \mu \mathrm{g} / \mathrm{mL})$ diluted in blocking buffer for $2 \mathrm{~h}$ at 4 ${ }^{\circ} \mathrm{C}$. Cells were washed and reacted with fluorescence-labeled goat anti-mouse IgG for $1 \mathrm{~h}$ at $4{ }^{\circ} \mathrm{C}$. Finally, cells were washed extensively in cold PBS and subjected to analysis by FACScan flow cytometer (BD Biosciences).

\section{ELISA-like PR3-binding assay}

Saturated mFc-fusion proteins (10 $\mu \mathrm{g} / \mathrm{mL})$, including CD177-mFc, GPR56-mFc, GPR97$\mathrm{mFc}$, and $\mathrm{mFc}$ were separately coated onto 96 -well plates at $4{ }^{\circ} \mathrm{C}$ for $16 \mathrm{~h}$. Coated plates were washed with the wash buffer (0.05\% Tween 20 in PBS) three times, and blocked with the blocking buffer ( $5 \%$ BSA in PBS) at $4{ }^{\circ} \mathrm{C}$ for $1 \mathrm{~h}$. Purified PR3 protein $\left(1,1.5,2\right.$, and $3 \mu \mathrm{g} / \mathrm{mL}$ in blocking buffer) was added at $4{ }^{\circ} \mathrm{C}$ for $2 \mathrm{~h}$. Wells were then washed extensively and incubated with the MCPR3-2 anti-PR3 mAb $(0.4 \mu \mathrm{g} / \mathrm{mL}$ in blocking buffer) at $4{ }^{\circ} \mathrm{C}$ for $2 \mathrm{~h}$. Wells were washed extensively and incubated with goat anti-mouse $\kappa$ chain HRP $(1 \mu \mathrm{g} / \mathrm{mL})$ at $4{ }^{\circ} \mathrm{C}$ for $1 \mathrm{~h}$. Following extensive washes, TMB (tetramethylbenzidine) substrate $\left(100 \mathrm{\mu L} /\right.$ well) was added at $4{ }^{\circ} \mathrm{C}$ for $20 \mathrm{~min}$ before adding the stop solution $\left(2 \mathrm{~N} \mathrm{H}_{2} \mathrm{SO}_{4}\right)(50 \mu \mathrm{L} /$ well). Reaction signals were detected by 
the ELISA reader at OD450.

\section{The ex vivo membrane PR3 activity assay}

The ex vivo neutrophil membrane PR3 activity was measured using the PR3-specific FRET substrate, Abz-VADnV-RDRQ-EDDNP (Cayman, MI, USA) exactly as described previously ${ }^{26}$. Briefly, freshly isolated $\mathrm{N} \varphi \mathrm{s}\left(1 \times 10^{6} /\right.$ well) were washed twice with PBS. Cells were then suspended in $150 \mu \mathrm{L}$ PBS and incubated with recombinant mFc-fusion proteins of interest in appropriate concentration for $10 \mathrm{~min}$ at $37^{\circ} \mathrm{C}$ before adding the PR3-specific FRET substrate $(20 \mu \mathrm{M})$. Fluorescence intensity was detected by the SpectraMax M2e ELISA reader (Molecular Devices, CA, USA) for at least 90 min during the reaction. When necessary, cells were treated with various protease inhibitors as indicated. The corresponding Vmax was analyzed by the Softmax Pro 5.3 software.

\section{The proximity-ligation assay (PLA)}

To detect the close interaction of CD177, PR3, GPR97, PAR2, and CD16b in human N 95 , the Duolink in situ Proximity Ligation Assay (PLA, Sigma-Aldrich ${ }^{\circledR}$, DU092013) was employed according to the manufacturer's protocol. Purified human N $\varphi s$ were cytospun on Poly-D-Lysine-coated coverslips and blocked in blocking buffer (1\% $\mathrm{BSA} / 5 \% \mathrm{NGS}$ in PBS) at $4^{\circ} \mathrm{C}$ for $60 \mathrm{~min}$. Cells were incubated with the receptor-specific Abs $(15 \mu \mathrm{g} / \mathrm{mL})$ pre-conjugated with the PLA-PLUS or -MINUS oligonucleotide probe (Sigma-Aldrich ${ }^{\circledR}$, DUO92009 and DU092010) at room temperature for $60 \mathrm{~min}$. The enzymatic ligation of the PLA-PLUS and-MINUS probes were performed at $37^{\circ} \mathrm{C}$ for 30 min, followed by circle amplification at $37^{\circ} \mathrm{C}$ for $90 \mathrm{~min}$ and finally incubated with Hoechst 33342 (Invitrogen) for nuclear staining. Cells were observed by the LSM780 confocal microscope system (ZEISS) at 40× magnification.

\section{Bacteria uptake and killing assays}

Freshly isolated $\mathrm{N} \varphi \mathrm{s}\left(1.25 \times 10^{6}\right.$ cells $\left./ \mathrm{mL}\right)$ were suspended in RPMI and incubated without or with GPR97 ${ }^{\mathrm{E}}-\mathrm{mFc}$ or control $\mathrm{mFc}$ fusion protein $(10 \mu \mathrm{g} / \mathrm{mL})$ respectively at $37^{\circ} \mathrm{C}$ for $30 \mathrm{~min}$. $\mathrm{N} \varphi s$ were then incubated with live E. coli (DH10B)(MOI 1:100), S. typhimurium (MOI 1:25), and S. aureus (MOI 1:100) in logarithmic-phase at $37^{\circ} \mathrm{C}$ for $1 \mathrm{hr}$. For the bacteria uptake assay, extracellular bacteria were removed by extensive washing in PBS, followed by incubation for 30 min in RPMI-1640 medium containing $50 \mu \mathrm{g} / \mathrm{mL}$ gentamicin. Afterwards, N $\varphi s$ were washed twice with PBS and lysed with 
$0.05 \%$ Triton $X-100$ in PBS. For the bacteria killing assay, $N \varphi s$ were collected and lysed with $0.05 \%$ Triton X-100 in PBS at desired time points after the bacteria uptake and the removal of extracellular bacteria by gentamicin. Serial dilutions of the $N \varphi$ lysate were plated on LB plates and incubated overnight at $37^{\circ} \mathrm{C}$ to determine the bacterial colonyforming units (CFU) the next day.

\section{Neutrophil-HUVEC co-culture}

Human umbilical vein endothelial cells (HUVECs) were maintained in Medium 199 supplemented with $10 \% \mathrm{FBS}$, L-glutamine, penicillin, streptomycin, $25 \mathrm{U} / \mathrm{mL}$ heparin and $30 \mu \mathrm{g} / \mathrm{mL}$ ECGS (endothelial cell growth supplement). HUVECs $\left(6 \times 10^{5}\right.$ cells/well) were plated on $1 \%$ gelatin-coated culture dishes $(6 \mathrm{~mm})$ until confluence. HUVECs were incubated with freshly isolated human $\mathrm{N} \varphi s\left(6 \times 10^{6}\right.$ cells/well) without or with indicated $\mathrm{mFc}$-fusion proteins $(10 \mu \mathrm{g} / \mathrm{mL})$ for 8 or $20 \mathrm{~h}$ at $37^{\circ} \mathrm{C}$. HUVECs incubated with LPS $(1 \mu \mathrm{g} / \mathrm{mL})$ were used as a positive control. Cells were washed with ice-cold PBS twice and collected for analysis using flow cytometry by staining with CD62E-APC (Eselectin, BD Pharmingen), CD54-APC (ICAM-1, BD Pharmingen), CD106-PE (VCAM-1, BD Pharmingen) and eNOS-PE (BD Pharmingen). For the endothelial cell permeability assay, HUVEC cells $\left(5 \times 10^{4}\right.$ cells/well) were plated on $1 \%$ gelatin-coated Transwell plates $(12 \mathrm{~mm})$ (Costar, Corning) containing polycarbonate membranes ( $3 \mu \mathrm{m}$ pore size) until confluence. Cells were incubated with freshly isolated human $\mathrm{N} \varphi s\left(1 \times 10^{6}\right.$ cells/well) without or with indicated $\mathrm{mFc}$-fusion proteins $(20 \mu \mathrm{g} / \mathrm{mL})$ for $24 \mathrm{~h}$. HUVECs incubated with thrombin $(20 \mathrm{nM})$ for $30 \mathrm{~min}$ were used as a positive control. Alternatively, HUVECs were incubated with heat-aggregated human $\lg G s(1 \mathrm{mg} / \mathrm{mL})$ without or with PAR2 antagonists (FSLLRY-NH ${ }_{2}$ and ENMD-1068)(100 $\left.\mu \mathrm{M}\right)$, PAR2 antibodies (SAM11 and MAB3949)(5 $\mu \mathrm{g} / \mathrm{mL})$, GPR97 antibodies (G97-A and BGP)(5 $\mu \mathrm{g} / \mathrm{mL}$ ) and mouse $\operatorname{lgG} 1$ as indicated for $8 \mathrm{~h}$. The lower chambers were replenished with fresh M199 growth medium while the upper chambers were filled with fresh medium containing $4 \%$ BSA and Evans blue $(0.67 \mathrm{mg} / \mathrm{mL})$. Cells were incubated for 30 $\min$ at $37^{\circ} \mathrm{C}$ and the optical density of the medium from lower chambers was measured at $650 \mathrm{~nm}$ in a spectrophotometer.

\section{Statistics}

All results were analyzed using GraphPad Prism (version 6.0; GraphPad Software, San Diego, CA, USA) and expressed as means \pm standard error of the mean (SEM) with the 
number of experimental replicates ( $\mathrm{n}$ ) provided. Differences between groups were determined by student's t-test, one-way and two-way ANOVA as indicated. In all cases, a probability $(p)$ value of $<0.05$ was accepted to reject the null hypothesis and considered as significant.

\section{Data availability}

All data needed to evaluate the conclusions in the paper are present in the main manuscript, the Supplementary Information and the Source data. Source data are provided with this paper and comprise all relevant raw data from each figure in the main manuscript and in the Supplementary Information. The Preliminary Full wwPDB X-ray Structure Validation Report of GPR97 extracellular domain (PDB ID 7QU8) (Deposition ID D_1292120102) has been assigned the following PDB DOI: https://doi.org/10.2210/pdb7QU8/pdb. The remaining raw data are available upon request from the corresponding author (H.H.L.) after signing confidentiality agreement.

\section{References:}

1. Mantovani A, Cassatella MA, Costantini C, Jaillon S. Neutrophils in the activation and regulation of innate and adaptive immunity. Nature reviews Immunology 11, 519-531 (2011).

2. Amulic B, Cazalet C, Hayes GL, Metzler KD, Zychlinsky A. Neutrophil function: from mechanisms to disease. Annual review of immunology 30, 459-489 (2012).

3. Korkmaz B, Horwitz MS, Jenne DE, Gauthier F. Neutrophil elastase, proteinase 3, and cathepsin $\mathrm{G}$ as therapeutic targets in human diseases. Pharmacological reviews 62, 726-759 (2010).

4. Pham CT. Neutrophil serine proteases: specific regulators of inflammation. Nature reviews Immunology 6, 541-550 (2006).

5. Kitching AR, et al. ANCA-associated vasculitis. Nature reviews Disease primers 6, 71 (2020).

6. von Vietinghoff $S$, et al. NB1 mediates surface expression of the ANCA antigen proteinase 3 on human neutrophils. Blood 109, 4487-4493 (2007).

7. Hu N, Westra J, Kallenberg CG. Membrane-bound proteinase 3 and its receptors: relevance for the pathogenesis of Wegener's Granulomatosis. Autoimmunity reviews 8, 510-514 (2009). 
8. Rarok AA, Stegeman CA, Limburg PC, Kallenberg CG. Neutrophil membrane expression of proteinase 3 (PR3) is related to relapse in PR3-ANCA-associated vasculitis. Journal of the American Society of Nephrology : JASN 13, 2232-2238 (2002).

9. Futosi K, Fodor S, Mocsai A. Neutrophil cell surface receptors and their intracellular signal transduction pathways. International immunopharmacology 17, 638-650 (2013).

10. Ramachandran R, Noorbakhsh F, Defea K, Hollenberg MD. Targeting proteinase-activated receptors: therapeutic potential and challenges. Nature reviews Drug discovery 11, 69-86 (2012).

11. Redecha P, Franzke CW, Ruf W, Mackman N, Girardi G. Neutrophil activation by the tissue factor/Factor VIla/PAR2 axis mediates fetal death in a mouse model of antiphospholipid syndrome. The Journal of clinical investigation 118, 34533461 (2008).

12. Shpacovitch V, Feld M, Hollenberg MD, Luger TA, Steinhoff M. Role of proteaseactivated receptors in inflammatory responses, innate and adaptive immunity. Journal of leukocyte biology 83, 1309-1322 (2008).

13. Shpacovitch V, Feld M, Bunnett NW, Steinhoff M. Protease-activated receptors: novel PARtners in innate immunity. Trends in immunology 28, 541-550 (2007).

14. Lin H, Trejo J. Transactivation of the PAR1-PAR2 heterodimer by thrombin elicits beta-arrestin-mediated endosomal signaling. The Journal of biological chemistry 288, 11203-11215 (2013).

15. Hamann J, et al. International Union of Basic and Clinical Pharmacology. XCIV. Adhesion G protein-coupled receptors. Pharmacological reviews 67, 338-367 (2015).

16. Schoneberg T, Liebscher I, Luo R, Monk KR, Piao X. Tethered agonists: a new mechanism underlying adhesion $G$ protein-coupled receptor activation. Journal of receptor and signal transduction research 35, 220-223 (2015).

17. Hamann J, Hsiao CC, Lee CS, Ravichandran KS, Lin HH. Adhesion GPCRs as Modulators of Immune Cell Function. Handbook of experimental pharmacology 234, 329-350 (2016).

18. Hsiao CC, et al. The Adhesion G Protein-Coupled Receptor GPR97/ADGRG3 Is Expressed in Human Granulocytes and Triggers Antimicrobial Effector Functions. Frontiers in immunology 9, 2830 (2018). 
19. Chiang NY, et al. Disease-associated GPR56 mutations cause bilateral frontoparietal polymicrogyria via multiple mechanisms. The Journal of biological chemistry 286, 14215-14225 (2011).

20. Beyrau M, Bodkin JV, Nourshargh S. Neutrophil heterogeneity in health and disease: a revitalized avenue in inflammation and immunity. Open biology 2, 120134 (2012).

21. Eulenberg-Gustavus C, Bahring S, Maass PG, Luft FC, Kettritz R. Gene silencing and a novel monoallelic expression pattern in distinct CD177 neutrophil subsets. The Journal of experimental medicine 214, 2089-2101 (2017).

22. Flesch BK, et al. Multicenter Study on Differential Human Neutrophil Antigen 2 Expression and Underlying Molecular Mechanisms. Transfusion medicine and hemotherapy : offizielles Organ der Deutschen Gesellschaft fur Transfusionsmedizin und Immunhamatologie 47, 385-395 (2020).

23. Clemmensen $\mathrm{SN}$, et al. Olfactomedin 4 defines a subset of human neutrophils. Journal of leukocyte biology 91, 495-500 (2012).

24. Jerke U, Marino SF, Daumke O, Kettritz R. Characterization of the CD177 interaction with the ANCA antigen proteinase 3. Scientific reports 7, 43328 (2017).

25. Korkmaz B, et al. Inhibitors and Antibody Fragments as Potential AntiInflammatory Therapeutics Targeting Neutrophil Proteinase 3 in Human Disease. Pharmacological reviews 68, 603-630 (2016).

26. Korkmaz B, et al. Measuring elastase, proteinase 3 and cathepsin $\mathrm{G}$ activities at the surface of human neutrophils with fluorescence resonance energy transfer substrates. Nature protocols 3, 991-1000 (2008).

27. Arac $D$, et al. A novel evolutionarily conserved domain of cell-adhesion GPCRs mediates autoproteolysis. The EMBO journal 31, 1364-1378 (2012).

28. Salzman GS, et al. Structural Basis for Regulation of GPR56/ADGRG1 by Its Alternatively Spliced Extracellular Domains. Neuron 91, 1292-1304 (2016).

29. Leon K, et al. Structural basis for adhesion G protein-coupled receptor Gpr126 function. Nature communications 11, 194 (2020).

30. David A, Fridlich R, Aviram I. The presence of membrane Proteinase 3 in neutrophil lipid rafts and its colocalization with FcgammaRIIIb and cytochrome b558. Experimental cell research 308, 156-165 (2005).

31. Jerke $U$, et al. Complement receptor Mac-1 is an adaptor for NB1 (CD177)- 
mediated PR3-ANCA neutrophil activation. The Journal of biological chemistry 286, 7070-7081 (2011).

32. Kim YC, et al. Membrane-bound proteinase 3 and PAR2 mediate phagocytosis of non-opsonized bacteria in human neutrophils. Molecular immunology 48, 1966-1974 (2011).

33. Kurosawa S, Esmon CT, Stearns-Kurosawa DJ. The soluble endothelial protein C receptor binds to activated neutrophils: involvement of proteinase-3 and CD11b/CD18. Journal of immunology 165, 4697-4703 (2000).

34. Granel J, et al. Pathogenicity of Proteinase 3-Anti-Neutrophil Cytoplasmic Antibody in Granulomatosis With Polyangiitis: Implications as Biomarker and Future Therapies. Frontiers in immunology 12, 571933 (2021).

35. Kruger $P$, et al. Neutrophils: Between host defence, immune modulation, and tissue injury. PLoS pathogens 11, e1004651 (2015).

36. Pawar NR, Buzza MS, Antalis TM. Membrane-Anchored Serine Proteases and Protease-Activated Receptor-2-Mediated Signaling: Co-Conspirators in Cancer Progression. Cancer research 79, 301-310 (2019).

37. Rothmeier AS, Ruf W. Protease-activated receptor 2 signaling in inflammation. Seminars in immunopathology 34, 133-149 (2012).

38. Camerer E, Huang W, Coughlin SR. Tissue factor- and factor X-dependent activation of protease-activated receptor 2 by factor VIla. Proceedings of the National Academy of Sciences of the United States of America 97, 5255-5260 (2000).

39. Le Gall SM, et al. Matriptase activation connects tissue factor-dependent coagulation initiation to epithelial proteolysis and signaling. Blood 127, 32603269 (2016).

40. Csernok $\mathrm{E}$, et al. Wegener autoantigen induces maturation of dendritic cells and licenses them for Th1 priming via the protease-activated receptor-2 pathway. Blood 107, 4440-4448 (2006).

41. Heuberger DM, Schuepbach RA. Protease-activated receptors (PARs): mechanisms of action and potential therapeutic modulators in PAR-driven inflammatory diseases. Thrombosis journal 17, 4 (2019).

42. Saffarzadeh $\mathrm{M}$, et al. Macrophage protease-activated receptor 2 regulates fetal liver erythropoiesis in mice. Blood advances 4, 5810-5824 (2020).

43. Cho C, Smallwood PM, Nathans J. Reck and Gpr124 Are Essential Receptor 
Cofactors for Wnt7a/Wnt7b-Specific Signaling in Mammalian CNS Angiogenesis and Blood-Brain Barrier Regulation. Neuron 95, 1221-1225 (2017).

44. Eubelen $\mathrm{M}$, et al. A molecular mechanism for Wnt ligand-specific signaling. Science 361, (2018).

45. Wang W, Qiao Y, Li Z. New Insights into Modes of GPCR Activation. Trends in pharmacological sciences 39, 367-386 (2018).

46. Cattaneo F, et al. Cell-surface receptors transactivation mediated by g proteincoupled receptors. International journal of molecular sciences 15, 1970019728 (2014).

47. Lin H, Liu AP, Smith TH, Trejo J. Cofactoring and dimerization of proteinaseactivated receptors. Pharmacological reviews 65, 1198-1213 (2013).

48. Schafer AE, Blaxall BC. G Protein Coupled Receptor-mediated Transactivation of Extracellular Proteases. Journal of cardiovascular pharmacology 70, 10-15 (2017).

49. Lin HH, Hsiao CC, Pabst C, Hebert J, Schoneberg T, Hamann J. Adhesion GPCRs in Regulating Immune Responses and Inflammation. Advances in immunology 136, 163-201 (2017).

50. Ping $Y Q$, et al. Structures of the glucocorticoid-bound adhesion receptor GPR97-Go complex. Nature 589, 620-626 (2021).

51. Ramachandran $\mathrm{R}$, et al. Neutrophil elastase acts as a biased agonist for proteinase-activated receptor-2 (PAR2). The Journal of biological chemistry 286, 24638-24648 (2011).

52. Nakayama $M$, et al. IL-32-PAR2 axis is an innate immunity sensor providing alternative signaling for LPS-TRIF axis. Scientific reports 3, 2960 (2013).

53. Korkmaz B, Kuhl A, Bayat B, Santoso S, Jenne DE. A hydrophobic patch on proteinase 3 , the target of autoantibodies in Wegener granulomatosis, mediates membrane binding via NB1 receptors. The Journal of biological chemistry 283, 35976-35982 (2008).

54. Wang JJ, et al. Gpr97 is essential for the follicular versus marginal zone Blymphocyte fate decision. Cell Death Dis 4, e853 (2013).

55. Shi J, et al. Gpr97 is dispensable for metabolic syndrome but is involved in macrophage inflammation in high-fat diet-induced obesity in mice. Scientific reports 6, 24649 (2016). 
56. Valtcheva N, Primorac A, Jurisic G, Hollmen M, Detmar M. The orphan adhesion G protein-coupled receptor GPR97 regulates migration of lymphatic endothelial cells via the small GTPases RhoA and Cdc42. The Journal of biological chemistry 288, 35736-35748 (2013).

57. Unkeless JC, Shen Z, Lin CW, DeBeus E. Function of human Fc gamma RIIA and Fc gamma RIIIB. Seminars in immunology 7, 37-44 (1995).

58. Robertson JG. Enzymes as a special class of therapeutic target: clinical drugs and modes of action. Current opinion in structural biology 17, 674-679 (2007).

59. Santos $\mathrm{R}$, et al. A comprehensive map of molecular drug targets. Nature reviews Drug discovery 16, 19-34 (2017).

60. Lin HH, Chang GW, Davies JQ, Stacey M, Harris J, Gordon S. Autocatalytic cleavage of the EMR2 receptor occurs at a conserved $G$ protein-coupled receptor proteolytic site motif. The Journal of biological chemistry 279 , 3182331832 (2004).

61. Aricescu AR, Lu W, Jones EY. A time- and cost-efficient system for high-level protein production in mammalian cells. Acta crystallographica Section D, Biological crystallography 62, 1243-1250 (2006).

62. Winter $\mathrm{G}$, et al. DIALS: implementation and evaluation of a new integration package. Acta crystallographica Section D, Structural biology 74, 85-97 (2018).

63. Evans PR, Murshudov GN. How good are my data and what is the resolution? Acta crystallographica Section D, Biological crystallography 69, 1204-1214 (2013).

64. Wagner A, Duman R, Henderson K, Mykhaylyk V. In-vacuum long-wavelength macromolecular crystallography. Acta crystallographica Section $D$, Structural biology 72, 430-439 (2016).

65. Vonrhein $\mathrm{C}$, et al. Data processing and analysis with the autoPROC toolbox. Acta crystallographica Section D, Biological crystallography 67, 293-302 (2011).

66. McCoy AJ. Solving structures of protein complexes by molecular replacement with Phaser. Acta crystallographica Section D, Biological crystallography 63, 3241 (2007).

67. Smart OS, et al. Exploiting structure similarity in refinement: automated NCS and target-structure restraints in BUSTER. Acta crystallographica Section D, Biological crystallography 68, 368-380 (2012).

68. Cowtan K. Recent developments in classical density modification. Acta 
crystallographica Section D, Biological crystallography 66, 470-478 (2010).

69. Thorn A, Sheldrick GM. ANODE: anomalous and heavy-atom density calculation. Journal of applied crystallography 44, 1285-1287 (2011).

70. Emsley P, Cowtan K. Coot: model-building tools for molecular graphics. Acta crystallographica Section D, Biological crystallography 60, 2126-2132 (2004).

\section{ACKNOWLEDGMENTS}

We thank Dr. Jörg Hamann and Dr. Gin-Wen Chang for their critical reading of the manuscript. The authors acknowledge the technical assistance from the Microscopy and Instrumentation Centers, Chang Gung University, Taoyuan, Taiwan. CZG was funded by the Wellcome Trust DPhil in Structural Biology programme. ES was funded by the Wellcome Trust (202827/Z/16/Z), the MRC (MR/L018039/1) and the EMBO Young Investigator programme. HHL was funded by Chang Gung Memorial Hospital (CMRPD1K0302, CMRPD1K0212, CMRPD1K0222, CMRPD1M0031, and CMRPD1K0132) and the Ministry of Science and Technology (MOST), Taiwan (MOST107-2320-B-182-006 and MOST-110-2320-B- 182-024).

\section{AUTHOR CONTRIBUTIONS}

T.Y.C., C. Z-G., K.Y.H., Y.C.C., Y.W.C., K.Y.I., Y.L.L., N.Y.C., H.Y.C., W.Y.T., C.Y.S., Y.M.W., Y.S.P., C.H.H., T.C.C., M.A., and S.R.H. performed experiments, M.S., S.G., C.H.L., and A.S. provided advice and critical reagents, T.Y.C., C. Z-G., E.S., and H.H.L. designed experiments and wrote the manuscript.

\section{COMPETING FINANCIAL INTERESTS}

The authors declare no competing financial interests. 
Fig. 1
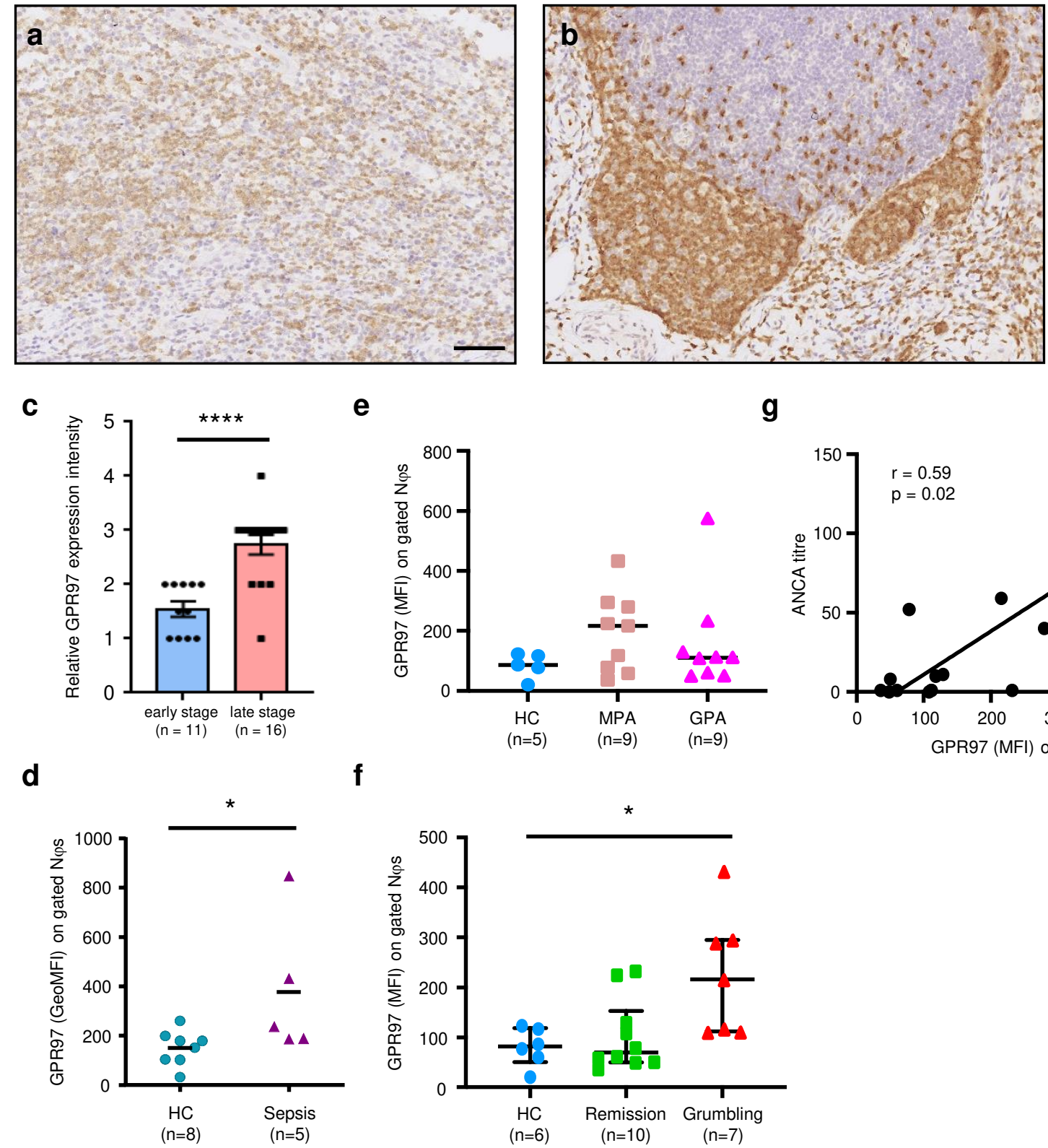

g
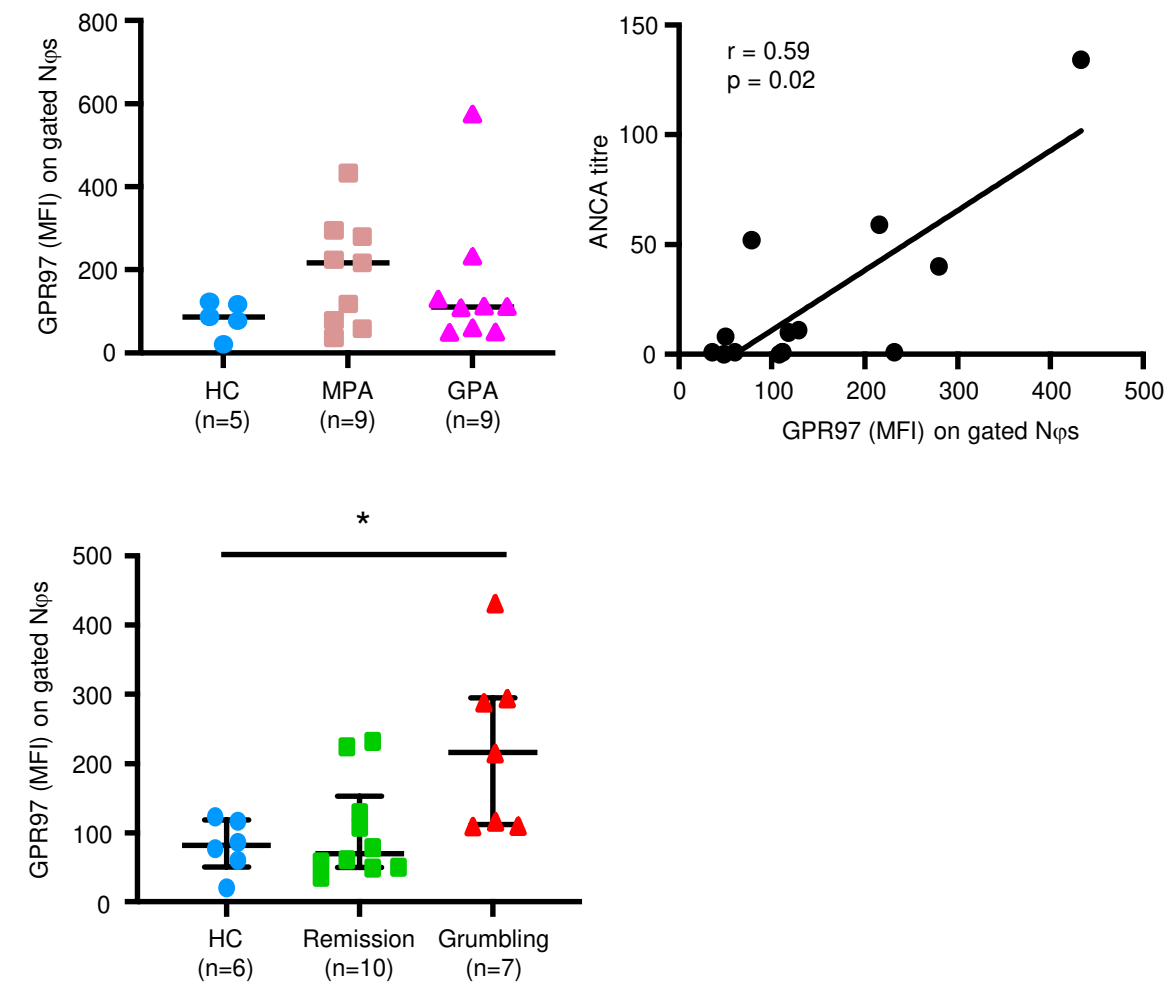
Figure legends:

Fig. 1 Up-regulated GPR97 expression in $\mathrm{N} \varphi s$ correlate with the disease status of inflammatory disorders. a, b. The immunohistochemical analyses of GPR97 expression in tissue sections of the early- $(n=11$, a) and late-stage $(n=16, b)$ appendicitis. Reactivity of the anti-GPR97 mAb to GPR97 Ag was shown as brownish staining. Scale bar, $100 \mu \mathrm{m}$. c. The relative GPR97 staining intensities in TIN $\varphi$ s of the early- and latestage appendicitis tissues. Data are means \pm SEM of at least 11 independent tissue samples of each experimental group (unpaired student's t-test). $* * * * p<0.0001$. d. Flow cytometry analyses of surface GPR97 levels of gated N $\varphi s$ of $H C(n=8)$ and bacterial sepsis patients $(n=5)$. e. The positive correlation of GPR97 levels with the ANCA titers of GPA and MPA patients. $\mathbf{f}$, g. Flow cytometry analyses of surface GPR97 levels of gated N $\varphi s$ of HC $(n=5)$ and patients of GPA $(n=9)$ and MPA $(n=9)$ based on the disease category (f) and status (g). Data are means \pm SEM of at least 5 independent samples of each patient group (unpaired student's t-test). ${ }^{*} p<0.05$. 
Fig. 2

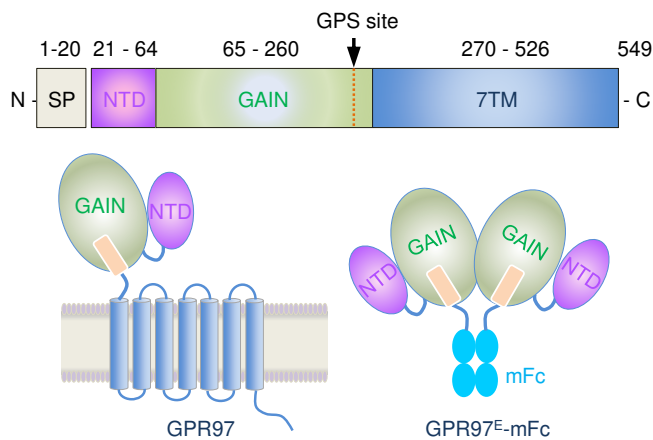

d

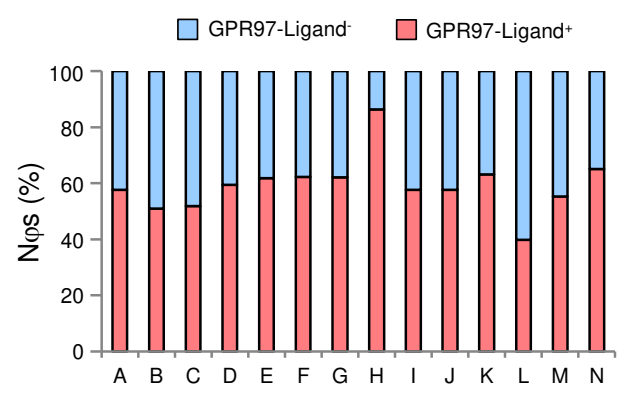

h
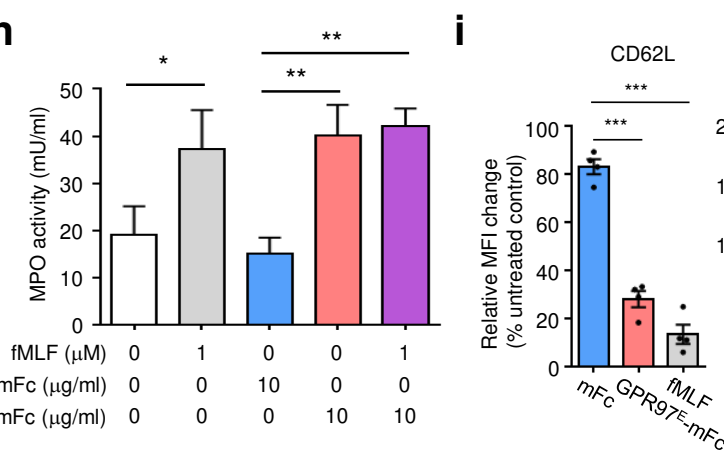

b

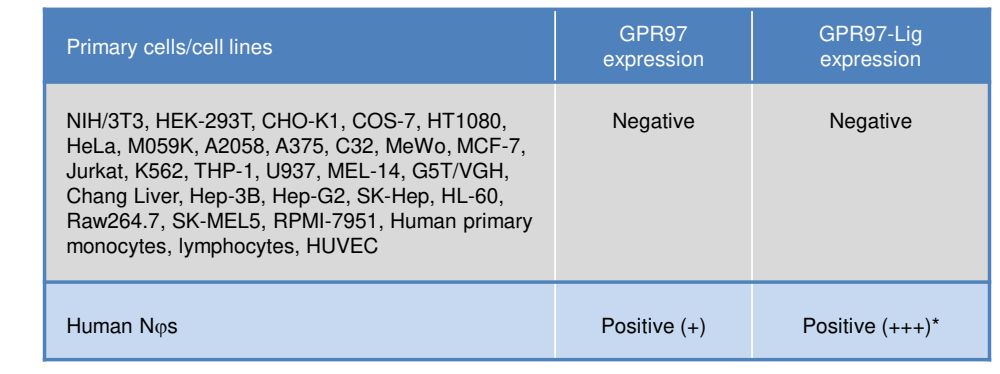

e

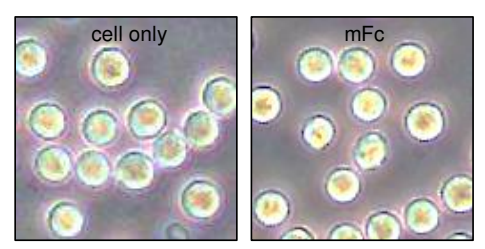

f
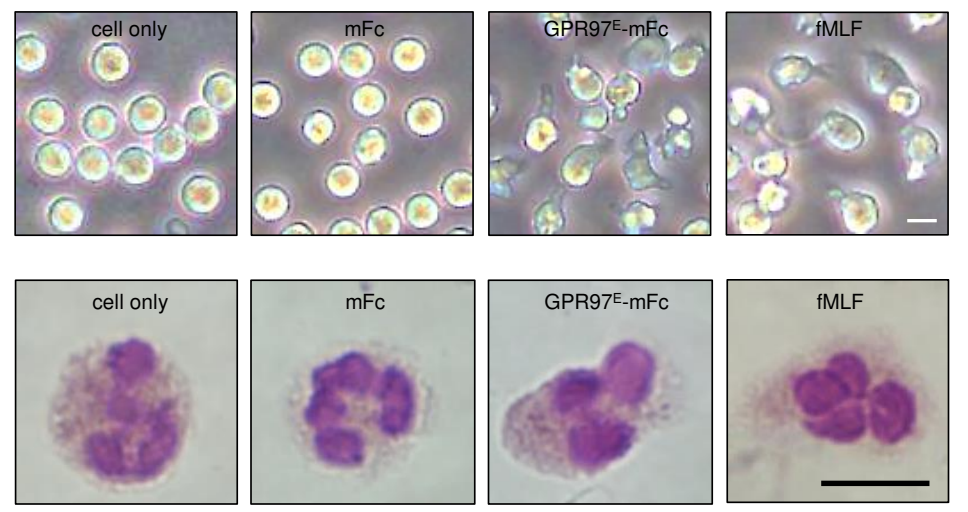

\section{g}
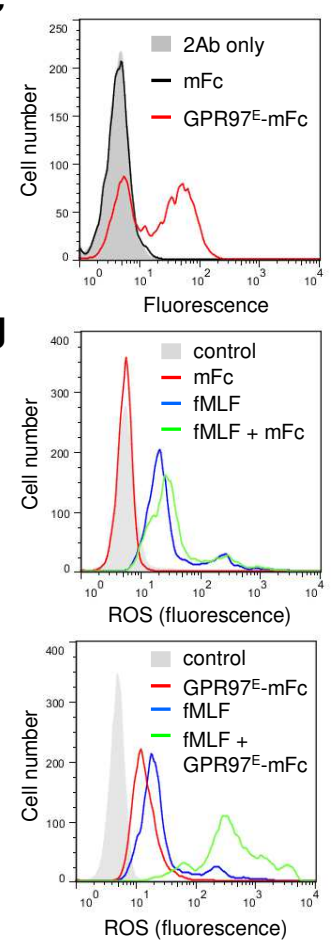

CXCR
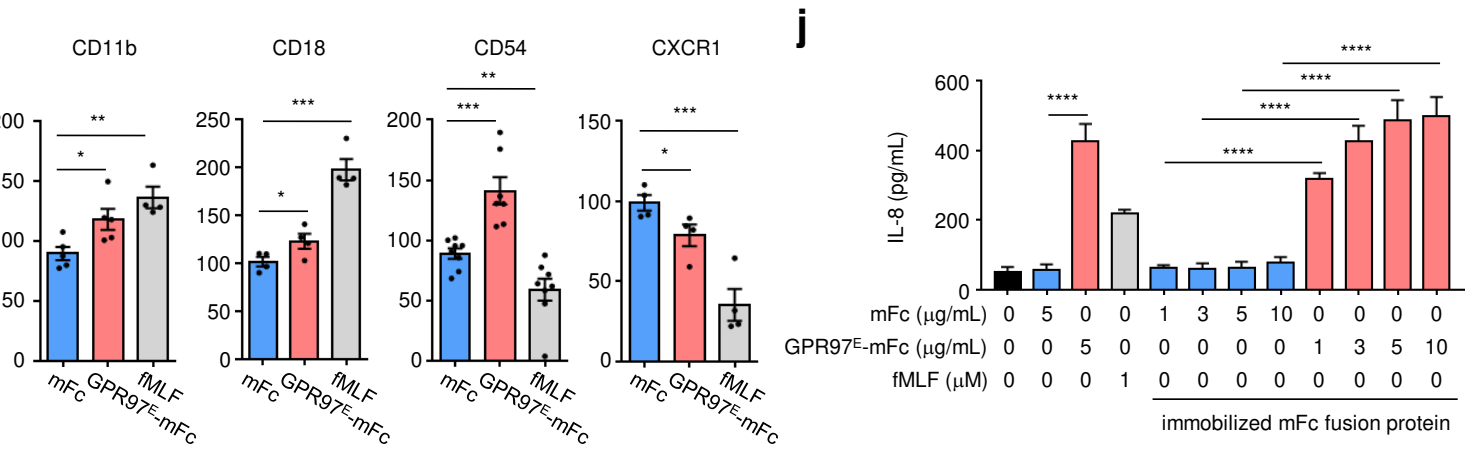
Fig. 2 Identification of a putative GPR97-ligand in a distinct human $\mathrm{N} \varphi$ subpopulation that is activated upon interaction with GPR97 ${ }^{\mathrm{E}}-\mathrm{mFc}$. a. Schematics of GPR97 receptor and GPR97 ${ }^{\mathrm{E}}-\mathrm{mFc}$ probe. b. List of cell samples screened for the expression of GPR97 and the putative GPR97-ligand. * indicates a subpopulation of primary human N $\varphi s$. c. A representative bimodal surface expression profile of the GPR97-ligand in human N $\varphi s$ detected by the GPR97 ${ }^{\mathrm{E}}-\mathrm{mFc}$ probe. d. Highly variable GPR97-ligand (blue) and GPR97-ligand ${ }^{+}$(pink) N $\varphi$ subsets in bloods of different donors (samples A to N). e, f. Morphological changes of $\mathrm{N} \varphi s$ induced by GPR97 ${ }^{\mathrm{E}}-\mathrm{mFc}$ as observed by inverted light microscope (e) and Wright-Giemsa stain (f). Scale bar, $20 \mu \mathrm{m}$. g-j. Phenotypic analyses of activated $\mathrm{N} \varphi s$ induced by GPR97 ${ }^{\mathrm{E}}-\mathrm{mFc}$ included up-regulated ROS production (g), MPO activity $(h, n=6)$, expressional changes of specific CD markers $(I, n=7$ for CD54 and $n=4$ for others) and IL-8 production $(j, n=3)$ of independent samples. The $\mathrm{mFc}$ protein and $\mathrm{AMLF}$ were used as a negative and a positive control, respectively. Data are presented as means \pm SEM in $\mathbf{h}, \mathbf{j}$ (two-way ANOVA) and $\mathbf{i}$ (one-way ANOVA). ${ }^{*} \mathrm{p}<$ $0.05 ; * * p<0.01 ; * * * p<0.001 ; * * * * p<0.0001$. 


\section{Fig. 3}

a

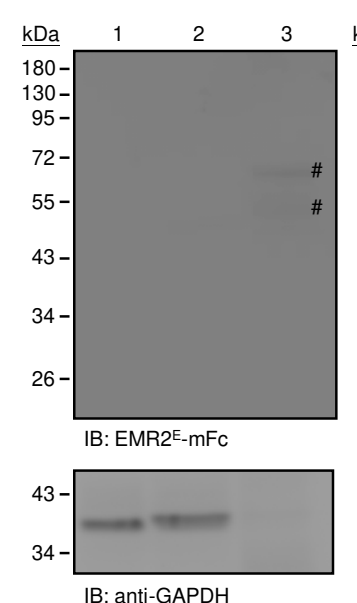

b

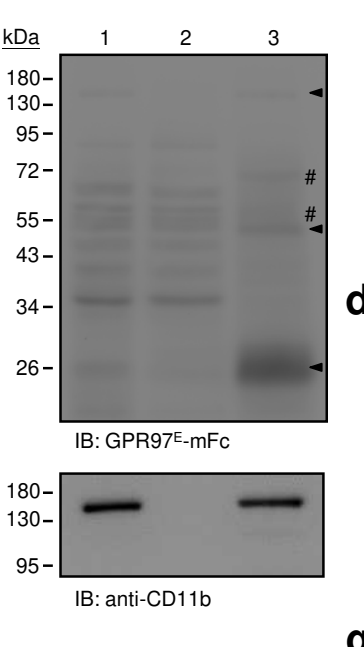

b
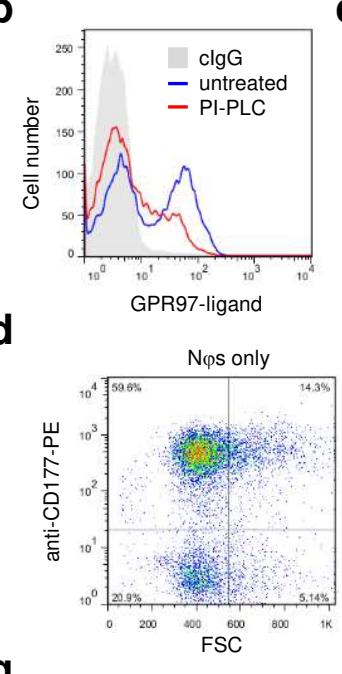

C
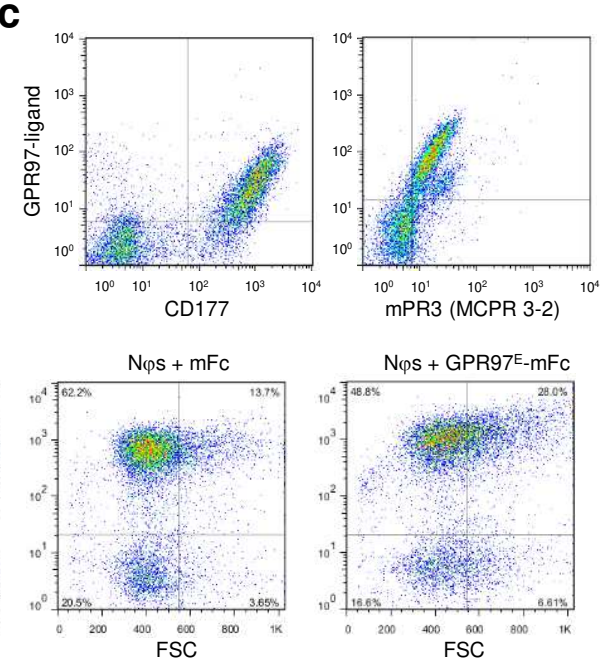

f

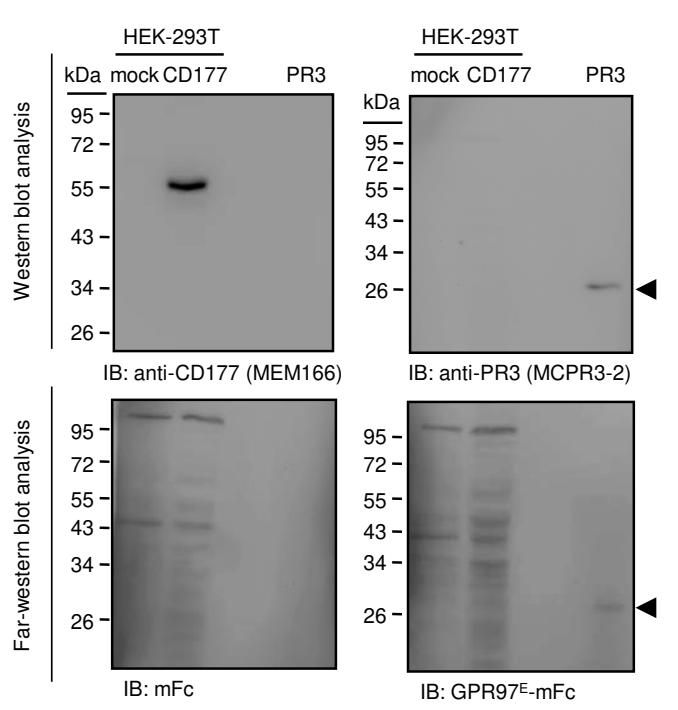

e

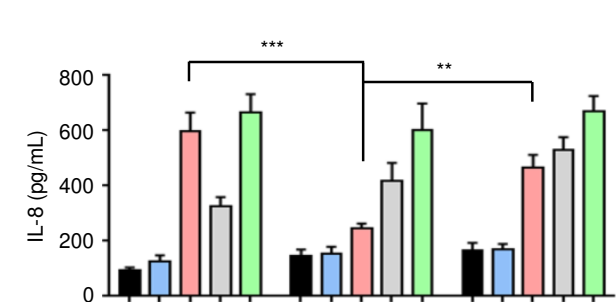

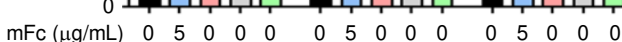

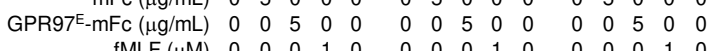

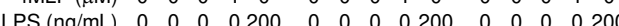
Unsorted $\mathrm{N} \varphi \mathrm{S} \frac{\mathrm{CD} 177^{-} \mathrm{N} \varphi \mathrm{S}}{\mathrm{CD} 177^{+} \mathrm{N} \varphi \mathrm{S}}$
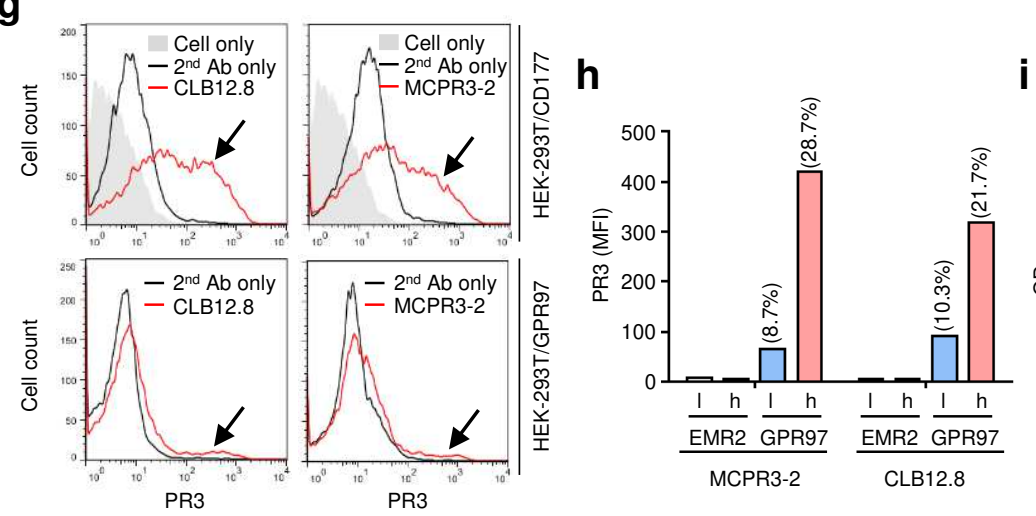

j

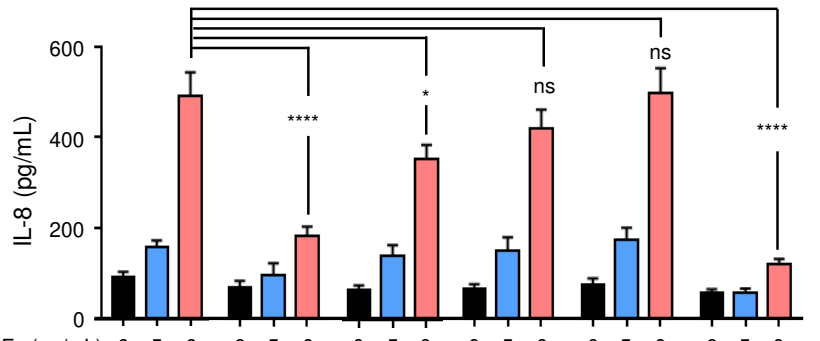

k

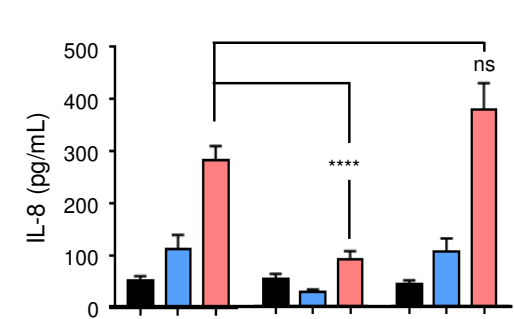

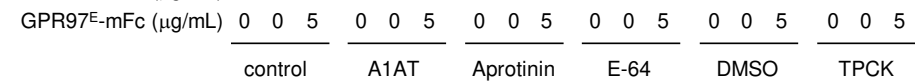

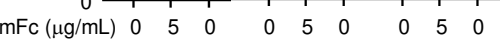

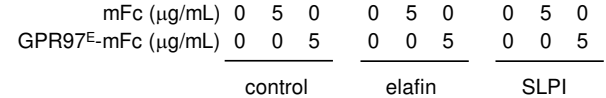

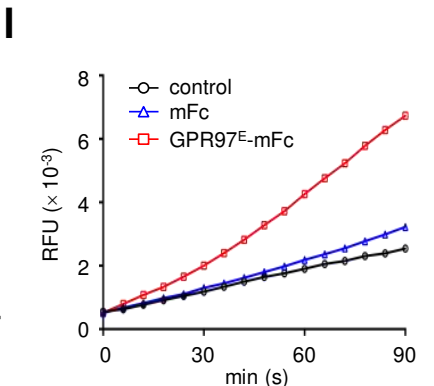

m

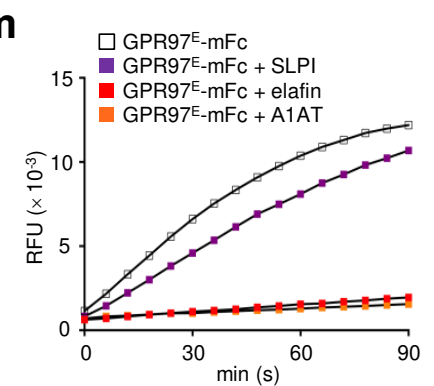

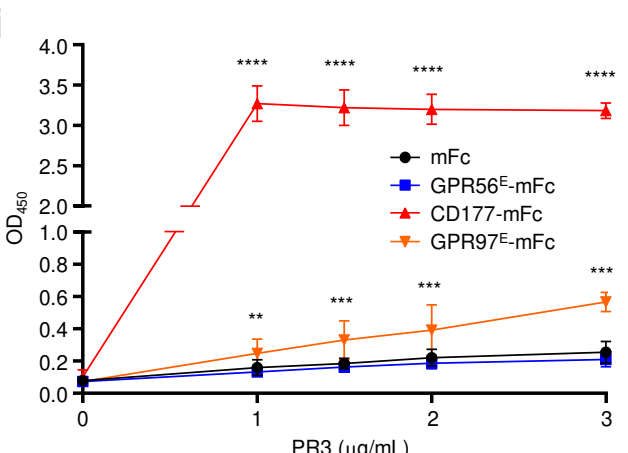

PR3 $(\mu \mathrm{g} / \mathrm{mL})$ 
Fig. 3 GPR97 is a binding partner and allosteric activator of mPR3. a. Far-western blot analyses of the putative GPR97-ligand(s)(black arrowheads). Lane 1, total lysate; Lane 2, cytoplasmic fraction; Lane 3, membrane fraction. The EMR2 $2^{\mathrm{E}}-\mathrm{mFc}$ protein was used as a negative control. Anti-CD11b and anti-GADPH Abs detected the corresponding protein markers of the membranous and cytoplasmic fractions, respectively. \#, nonspecific signals. b. FACS-based ligand-binding analysis of $\mathrm{N} \varphi s$ pre-treated without or with PI-PLC. c. Dot-plots of N $\varphi$ s double-stained with GPR97 ${ }^{\mathrm{E}}-\mathrm{mFc}$ and anti-CD177 or anti-PR3 mAb. d. Dot-plots (FSC vs. surface CD177 levels) of $N \varphi s$ incubated with GPR97E-mFc. e. IL-8 produced by unsorted and sorted N $\varphi$ s incubated with various reagents as indicated. $n=5$ independent experiments. Cells treated with $\mathrm{fMLF}$ and LPS were included as positive controls. f. Far-western blot analysis of specific GPR97-PR3 binding. Samples included total lysates of mock- and CD177-transfected HEK-293T cells as well as purified PR3. The arrowheads indicated the specific signals detected by the anti-PR3 mAb and the GPR97 ${ }^{\mathrm{E}}-\mathrm{mFc}$ probe. $\mathbf{g}$, $\mathbf{h}$. The FACS-based PR3-binding assay of unsorted (g) and FACS-sorted (h) HEK-293T cells expressing CD177 or GPR97. The black arrows in (g) indicated the specific PR3-binding. FACS-sorted GPR97 ${ }^{\text {high }}(\mathrm{h})$ and GPR97 $^{\text {low }}$ (I) HEK-293T cells were examined. Numbers on top of the bars indicated the percentage of PR3-binding cell populations. i. ELISA-like PR3-binding analyses. CD177$\mathrm{mFc}$ and GPR56-mFc proteins were included as the positive and negative controls, respectively. $n=6$ biologically independent samples. $\mathbf{j}$, k. ELISA analysis of IL-8 secreted by $N \varphi s$ treated without or with indicated protease inhibitors. $n=5(\mathbf{j}), 4$ (k) independent experiments. Data are presented as means \pm SEM in e, i-k (two-way ANOVA). ${ }^{*} \mathrm{p}<0.05 ;{ }^{*} \mathrm{p}<0.01 ;{ }^{* *} \mathrm{p}<0.001 ;{ }^{* * *} \mathrm{p}<0.0001 ; \mathrm{ns}$, non-significant. I, $\mathrm{m}$. The ex vivo mPR3 enzymatic activity of $N \varphi s$ incubated in the absence or presence of $\mathrm{mFc}$ or GPR97-mFc protein probe without or with protease inhibitors as indicated. 
Fig. 4

a
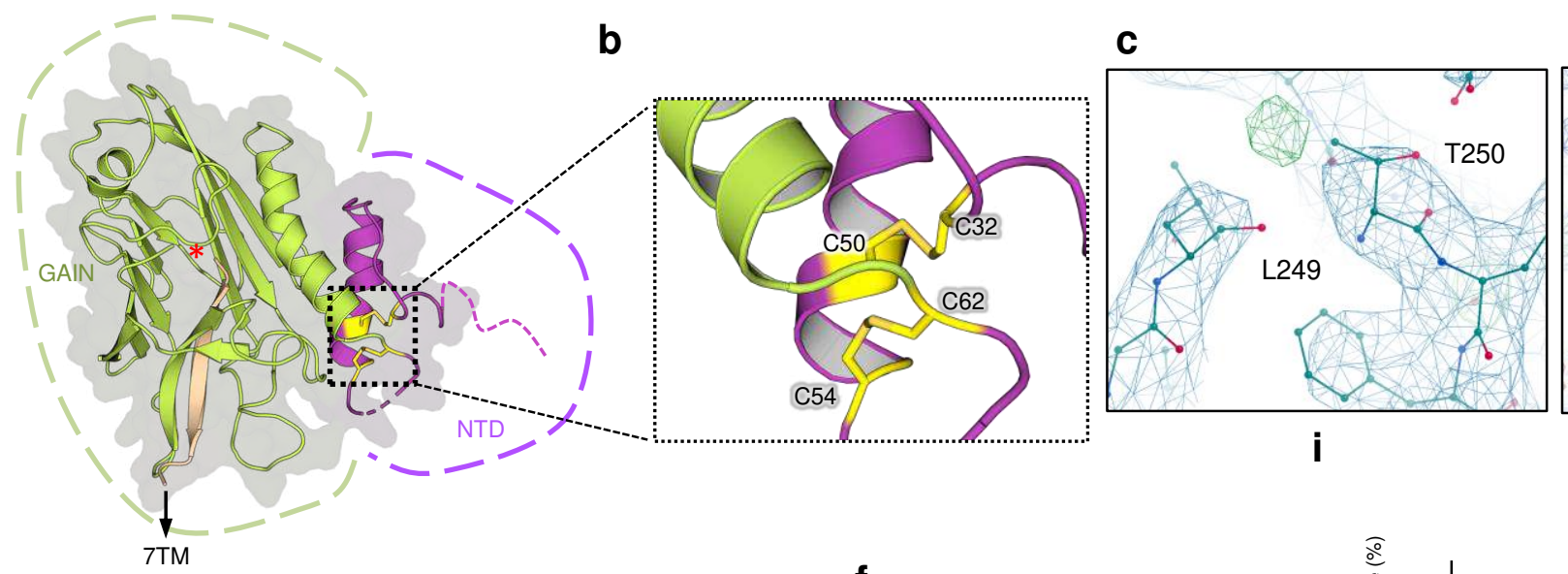

d

e
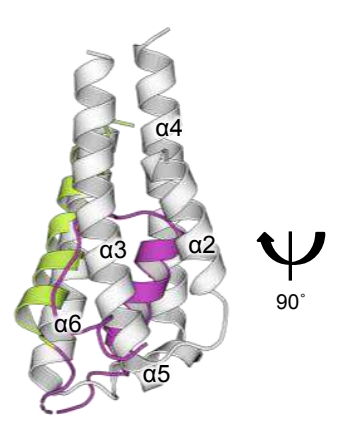

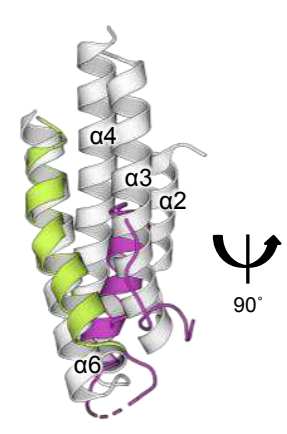

g

\begin{tabular}{ll} 
& \multicolumn{2}{c}{ GPS } \\
GPR97E-mFc NTD GAIN $\mathrm{mFc}$
\end{tabular} GPR97 ${ }^{\text {NTD/GPR56 GAIN_mFc }}$ NTD GAIN $\quad \mathrm{mFc}$

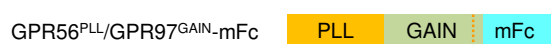
\begin{tabular}{l|l|l|l} 
GPR56E-mFc & PLL & GAIN & $\mathrm{mFc}$
\end{tabular}

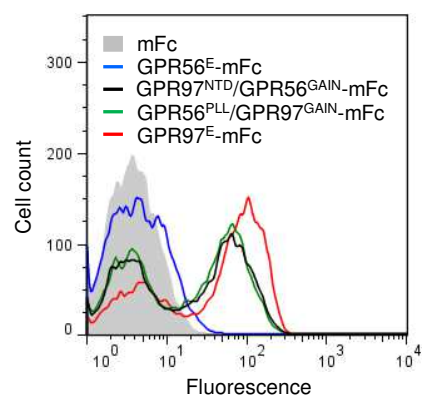

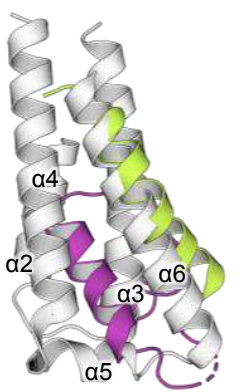

h
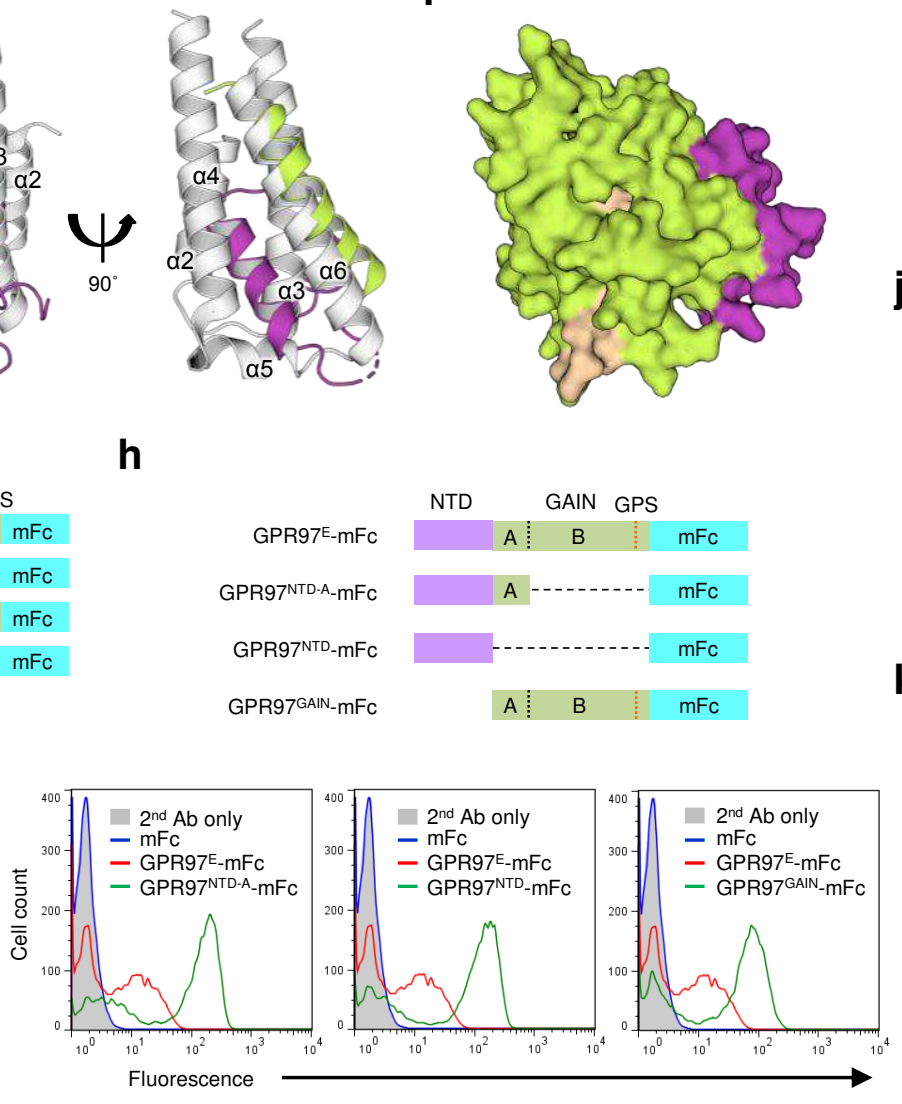

GPR97 ${ }^{\mathrm{NTD}}-\mathrm{mFc}$ GPR97 ${ }^{\text {GAIN-}_{-} \mathrm{mFC}}$

\begin{tabular}{l:l|l}
$\mathrm{A}$ & $\mathrm{B}$ & $\mathrm{mFc}$
\end{tabular}

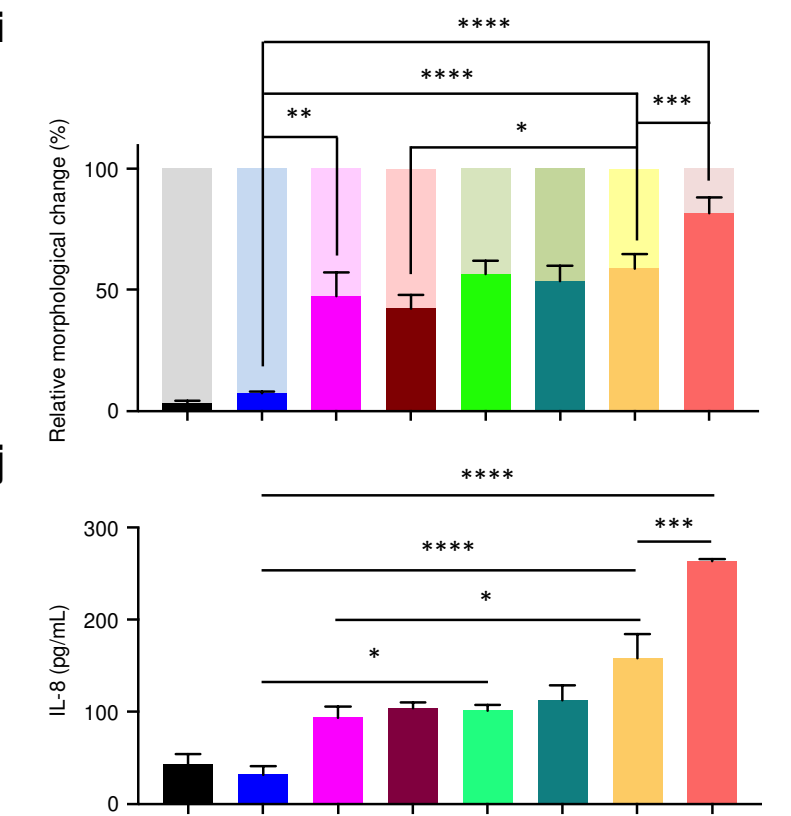

k

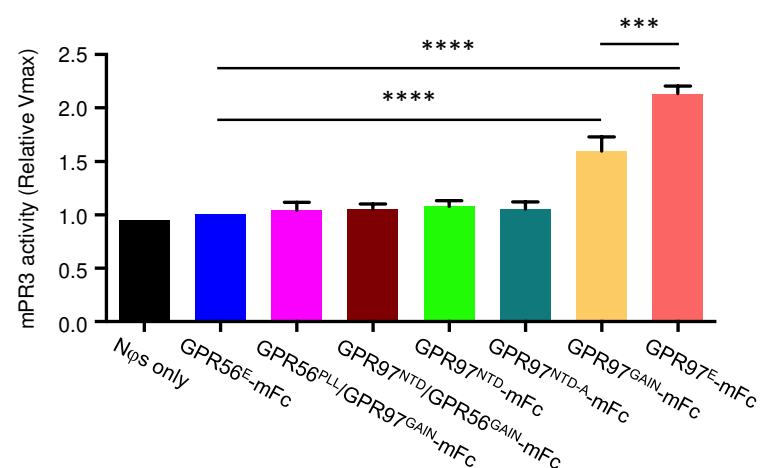


Fig. 4 Structural analysis of GPR97-ECR and mapping of the mPR3-binding regions. a. Cartoon representation of the GPR97-ECR structure obtained from X-ray crystallography experiments. The NTD is depicted in purple and the GAIN domain in green and beige (the $13^{\text {th }} \beta$-strand). Cysteine residues involved in linking and stabilising the NTD are shown as yellow sticks. The red asterisk indicates the GPS cleavage site. $\mathbf{b}$. Close-up of the two disulphide bridges, C32-C50 and C54-C62, that are involved in the stabilisation of the NTD and GAIN domains. c, d. 2Fo-Fc electron density maps are shown at the 1 sigma level in blue. Fo-Fc electron density maps are shown at $+/-3$ sigma level in green and red, respectively. (c) Stick representation of the GPR97 model in a 2Fo-Fc electron density map after several rounds of refinement. The presence of a small green electron density between L249 and T250 suggests the existence of a mixture of autoproteolysed and non-proteolysed forms within the crystal. (d) The $\alpha$ helix of GPR97 GAIN subdomain A is depicted in a red trace in a 2Fo-Fc electron density map. Clear additional density is seen on top of the GPR97 GAIN domain, accounting for the small NTD. e. Structural alignment of the $\alpha$ helix from the GPR97 GAIN domain (green) with the $\alpha 2-\alpha 6$ helices from LPHN1 GAIN domain (white, PDB ID: 4DLQ) showing that the NTD (purple) is not part of the GAIN domain. f. Surface representation of GPR97-ECR. $\mathbf{g}$, h. Mapping of the mPR3-binding region(s) using the domain-swapped (g) and domain-truncated (h) GPR97-mFc probes as indicated. $n=5$ (g) and 6 (h) independent experiments. i-k. Activated $N \varphi$ phenotypes, namely morphological changes (i), IL-8 production (j), and the ex vivo mPR3 enzymatic activity (k) treated with various GPR97-mFc probes as indicated. $n=3(\mathbf{i}), 4(\mathbf{j})$, and $6(\mathbf{k})$ independent experiments. The bright and dim colors in (i) represent the percentage of irregular-shaped and round-shaped $N \varphi s$, respectively. The results shown in (k) are relative Vmax of the ex vivo mPR3 enzymatic activity. Data are presented as means \pm SEM (two-way ANOVA). ${ }^{*} p<0.05 ;{ }^{* *} p<0.01 ; * * p<0.001 ; * * * p<0.0001$. 
Fig. 5

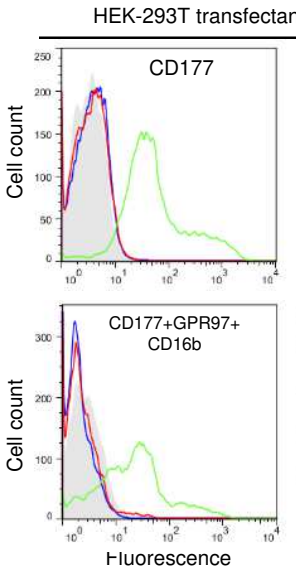

d

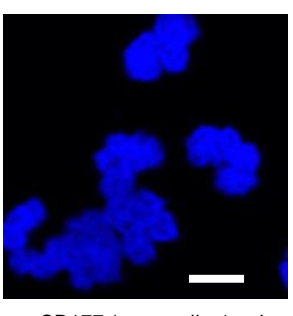

$\alpha$-CD177 (sense oligo) only

$\alpha$-CD177 (sense oligo) + $\alpha$-PR3 (anti-sense oligo)

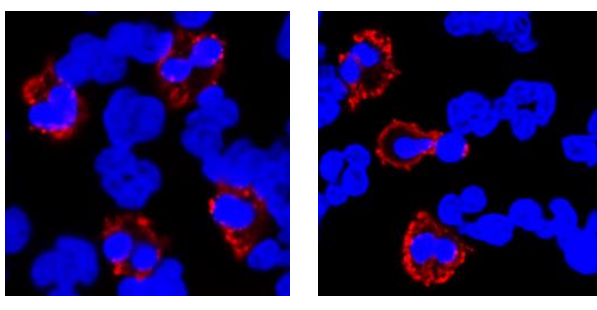

$\alpha$-CD177 (sense oligo) + $\alpha$-GPR97 (anti-sense oligo)

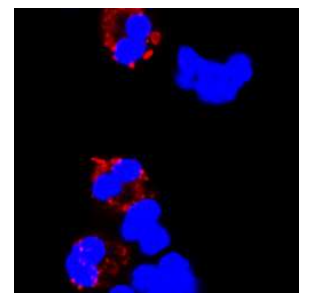

$\alpha$-CD177 (sense oligo) $\alpha-$ PAR2 (anti-sense oligo)

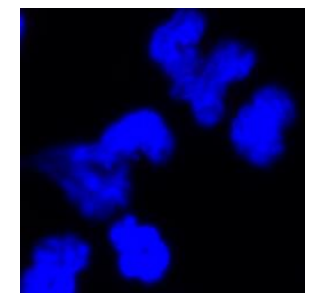

a-CD177 (sense oligo) +

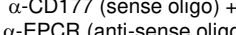
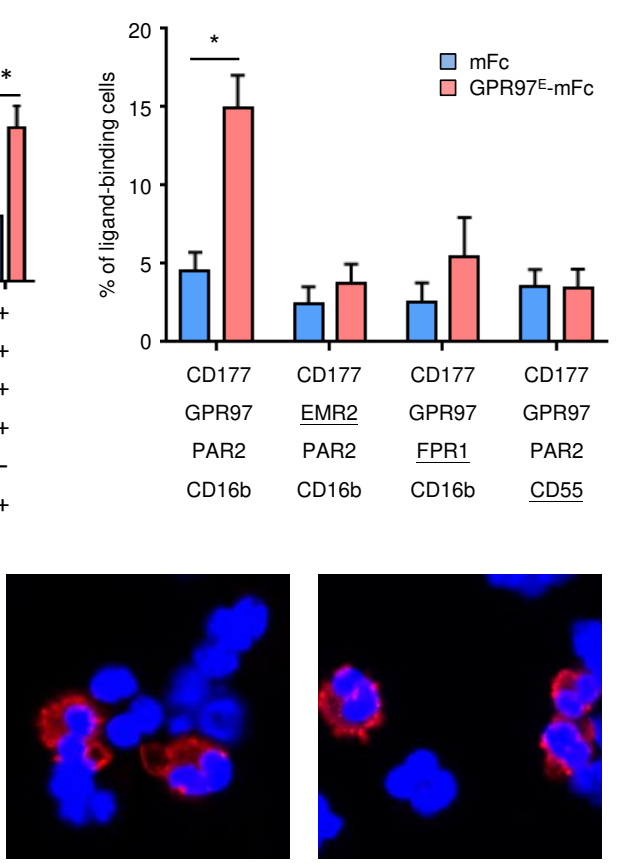

$\alpha$-CD16b (sense oligo) + $\alpha$-GPR97 (anti-sense oligo)

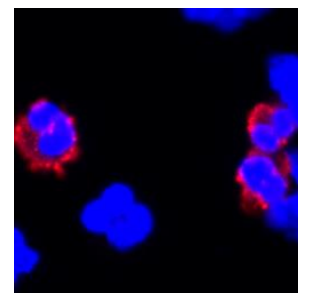

$\alpha$-CD16b (sense oligo) + $\alpha$-PAR2 (anti-sense oligo)
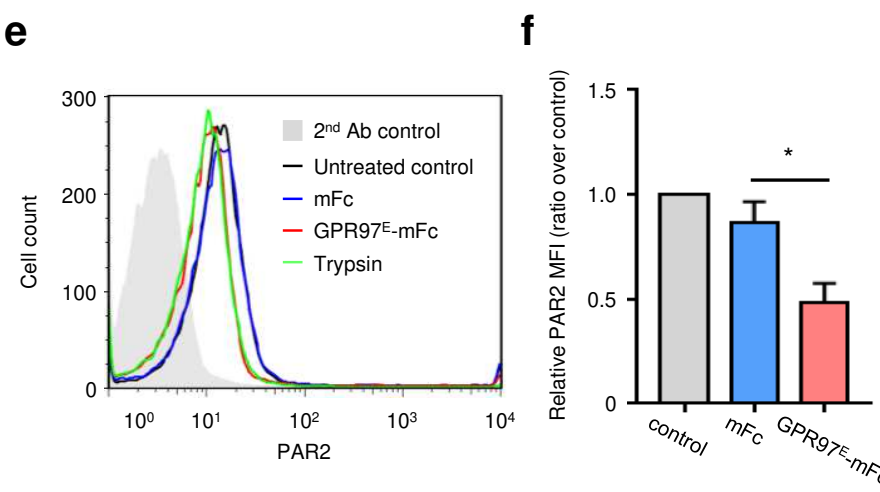

g

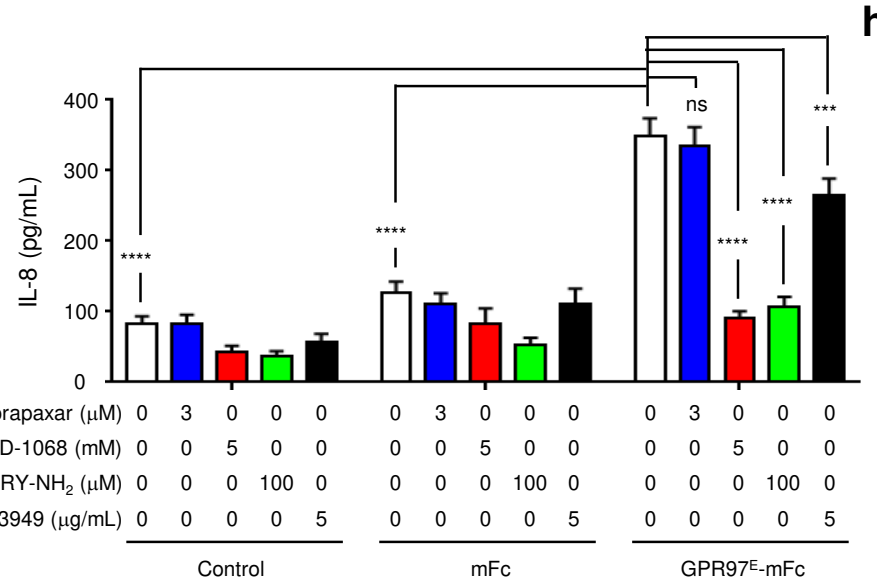

h

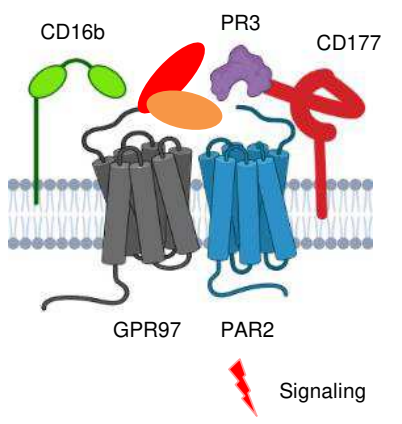

$\mathrm{N} \varphi$ inflammatory activation 
Fig. 5 The CD177/GPR97/PAR2/CD16b receptor interactome is required for efficient GPR97-mPR3 interaction that induces PAR2 transactivation. a-c. Flow cytometry analyses of GPR97-mPR3 interaction in HEK-293T cells expressing various receptors as indicated. (a) Surface levels of membrane-bound PR3 (green), GPR97 E-mFc (red), and $\mathrm{mFc}$ (blue) were detected using appropriate Abs. (b, c) The percentage of transfectants displaying the positive GPR97 ${ }^{\mathrm{E}}-\mathrm{mFc}$ binding signal in the ligand-binding assay. $\mathrm{n}=3(\mathbf{b})$, 4 (c) independent experiments. The underlined receptor in (c) indicates the substituted component of the core receptor complex. Data are presented as means \pm SEM (unpaired student's t-test). ${ }^{*} p<0.05$. $d$. The PLA analyses showed the interaction signals of specific receptor pairs of the CD177-associated interactome in resting N $\varphi s$. Scale bar, $10 \mu \mathrm{m}$. e, f. Flow cytometry analyses of PAR2 proteolysis in $\mathrm{N} \varphi s(\mathbf{e})$ and HEK293T cells expressing the CD177-associated receptor complex (f). Trypsin-treated N $\varphi s$ and $\mathrm{mFc}$ were included as a positive and a negative control, respectively. $\mathrm{n}=5$ independent experiments. Data are presented as means \pm SEM (one-way ANOVA). ${ }^{*} p$ $<0.05$. g. ELISA analyses of IL-8 secreted by $N \varphi s$ incubated with the indicated reagents. $\mathrm{n}=4$ independent experiments. Data are presented as means \pm SEM (two-way ANOVA). ${ }^{* * *} \mathrm{p}<0.001 ; * * *{ }_{\mathrm{p}}<0.0001 ; \mathrm{ns}$, non-significant. $\mathrm{h}$. Schematic diagram of the PR3/CD177/GPR97/PAR2/CD16b interactome in N $\varphi s$. Created using Biorender. 


\section{Fig. 6}

a

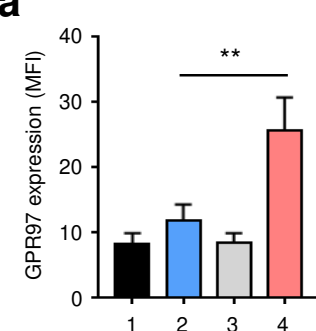

b

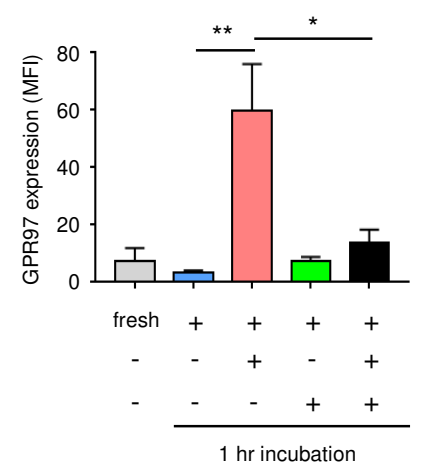

C

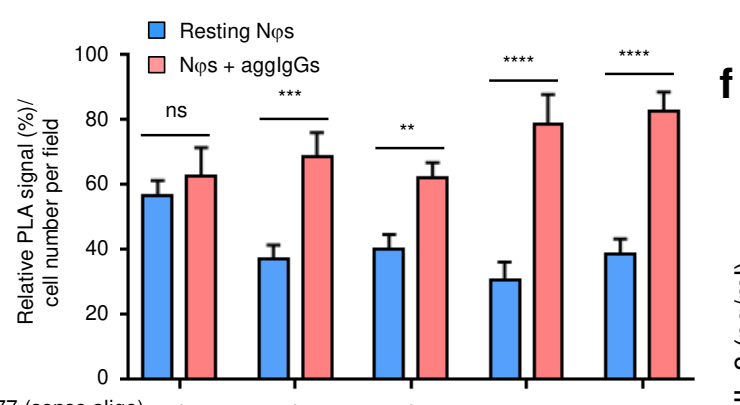

$\alpha$-CD177 (sense oligo) + $\alpha$-PR3 (anti-sense oligo) + $\alpha$-GPR97 (anti-sense oligo)

$\alpha$-PAR2 (anti-sense oligo)

$\alpha$-CD16b (sense oligo)

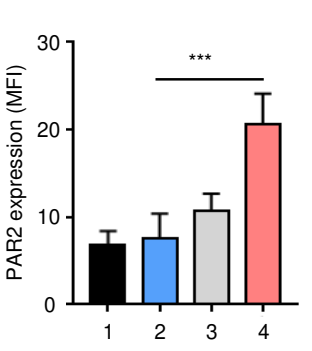

d
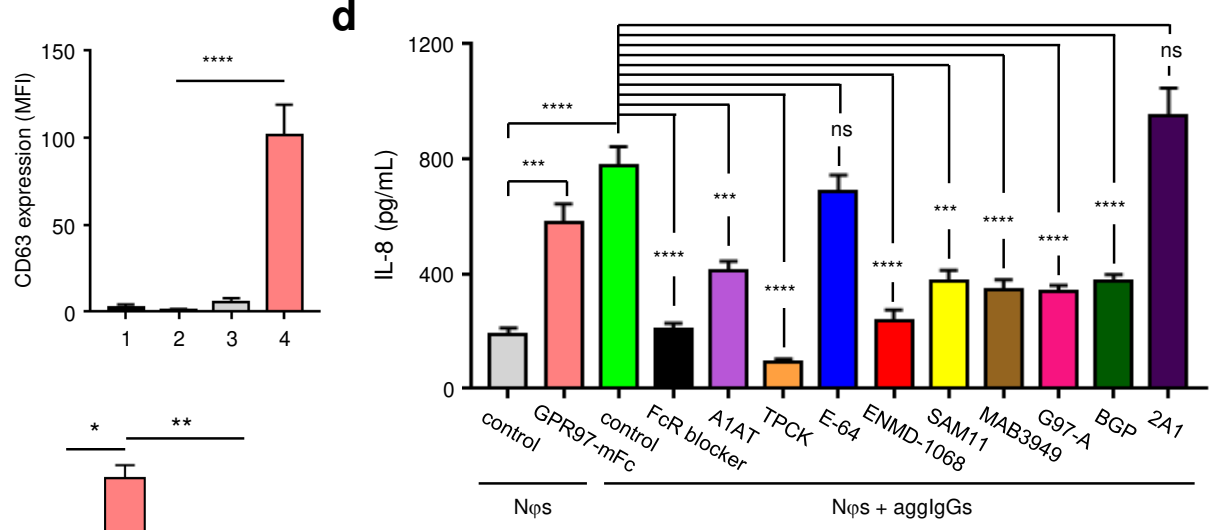

e
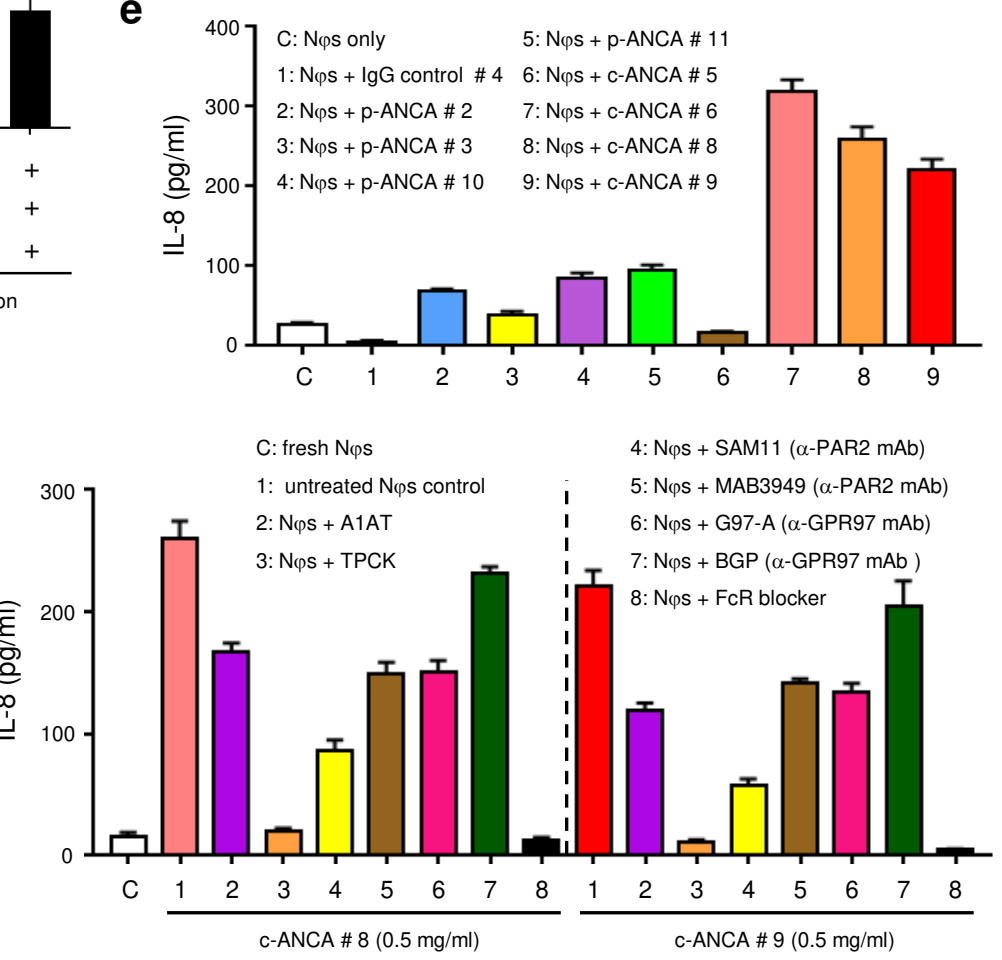
Fig. 6 Up-regulated GPR97 and PAR2 expression in activated N $\varphi s$ induces GPR97PAR2 transactivation. a, b. Flow cytometry analyses of GPR97 and PAR2 expression in resting $\mathrm{N} \varphi s$ treated without or with the azurophil granule degranulation stimulants (a) and heat-aggregated IgGs (algGs) (b) as indicated. (a) Samples include: 1, fresh N $\varphi s ; 2$,

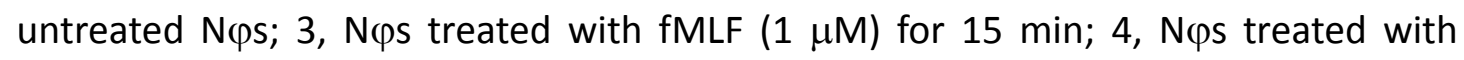
cytochalasin $\mathrm{B}$ (cytoB, $5 \mu \mathrm{M}$ ) for $5 \mathrm{~min}$, followed by fMLF $(1 \mu \mathrm{M})$ for $10 \mathrm{~min}$. CD63 expression was used as a degranulation marker of azurophil granule. c. Relative PLA signals of indicated receptor pairs in resting and algG-treated $N \varphi s$. d. ELISA analyses of IL- 8 secreted by $N \varphi s$ incubated at $37^{\circ} \mathrm{C}$ for $3 \mathrm{hr}$ as indicated. Data are presented as means \pm SEM in a-d (two-way ANOVA). $\mathrm{n}=3$ or 4 independent experiments. ${ }^{* * *} \mathrm{p}<$ $0.001 ; * * * * p<0.0001$; ns, non-significant. e, f. ELISA analyses of IL-8 secreted by N $\varphi s$ that were incubated with purified IgGs $(0.5 \mathrm{mg} / \mathrm{ml})$ from different PR3-ANCA and MPO-ANCA patients at $37{ }^{\circ} \mathrm{C}$ for $3 \mathrm{hr}$ as indicated. Data shown were results of one representative experiment. ENMD-1068: PAR2 antagonist; SAM11 and MAB3949: antiPAR2 mAbs; G97-A and BGP: anti-GPR97 mAbs; 2A1: anti-EMR2 mAb. 


\section{Fig. 7}

a

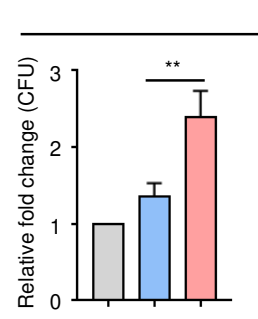

$\begin{array}{lll}0 & 10 & 0\end{array}$

\begin{tabular}{rrr}
0 & 0 & 10 \\
\hline$N P S / E . c o l i$
\end{tabular}

$\mathrm{N} \varphi \mathrm{s} / \mathrm{E}$. coli

$(\mathrm{N}=7)$

C

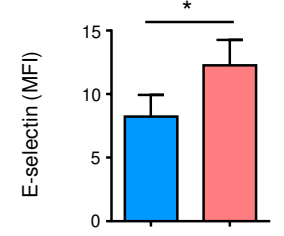

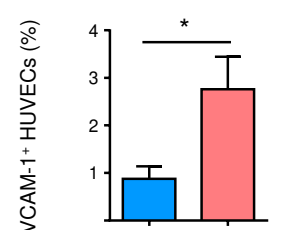
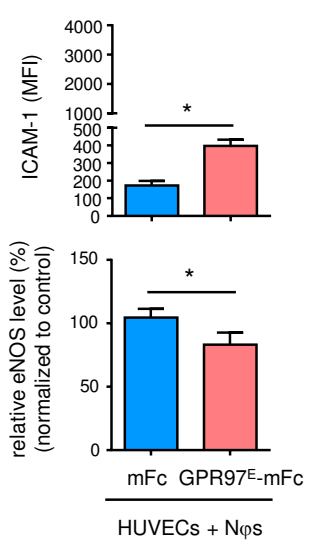

Bacteria uptake assay

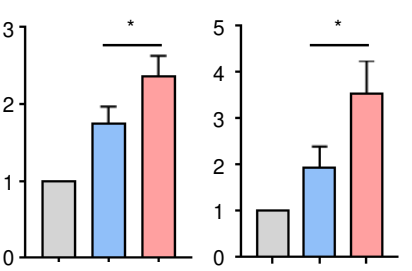

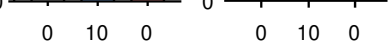

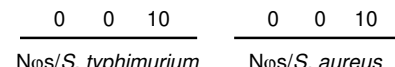

$(1 / 25)$
$(N=13)$

$(\mathrm{N}=8)$ b

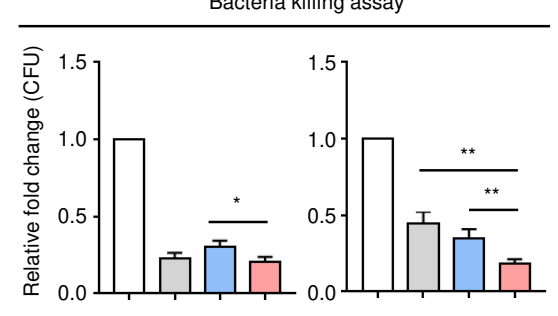

$\mathrm{mFc}(\mu \mathrm{g} / \mathrm{mL})$

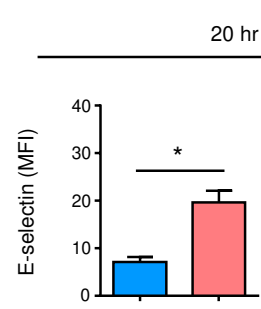

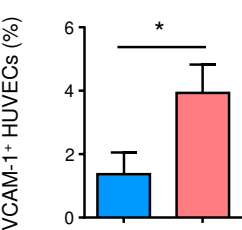
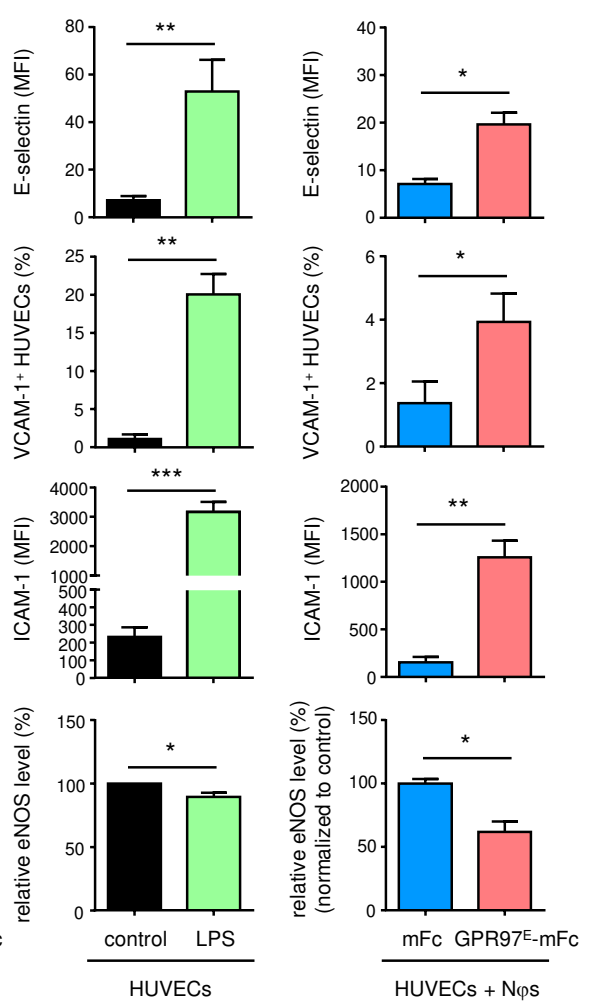

$\mathrm{N} \varphi \mathrm{S} / \mathrm{S}$. typhimurium (1/25) N $\mathrm{N} / \mathrm{S}$. aureus $(1 / 100)$

Incubation: $\overline{0 \mathrm{hr}} \frac{(\mathrm{N}=11)}{2.0 \mathrm{hr}} \quad \overline{\mathrm{hr}} \frac{(\mathrm{N}=11)}{0.5 \mathrm{hr}}$

$\mathrm{hr}$ incubation
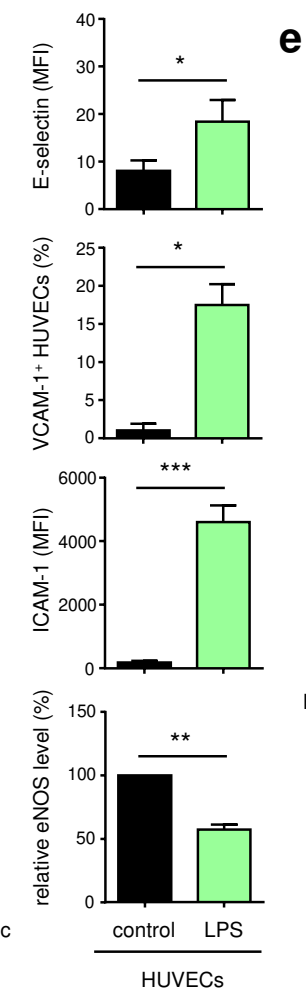

d

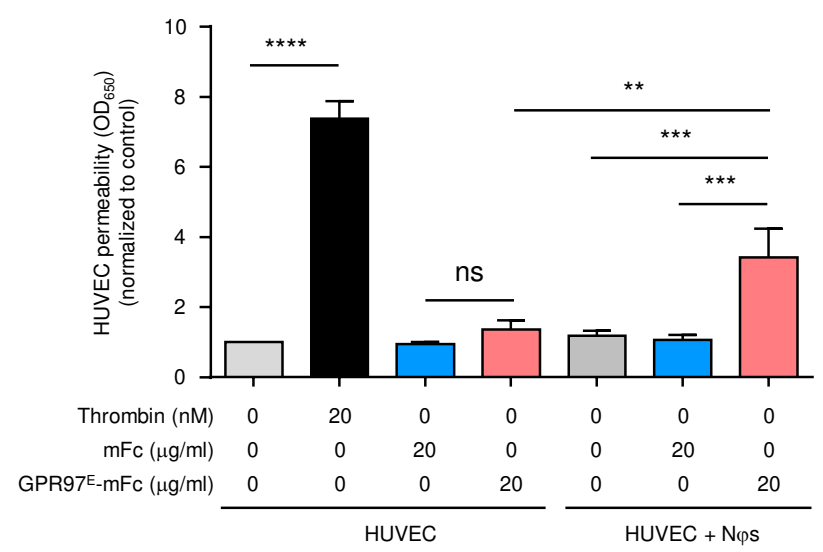

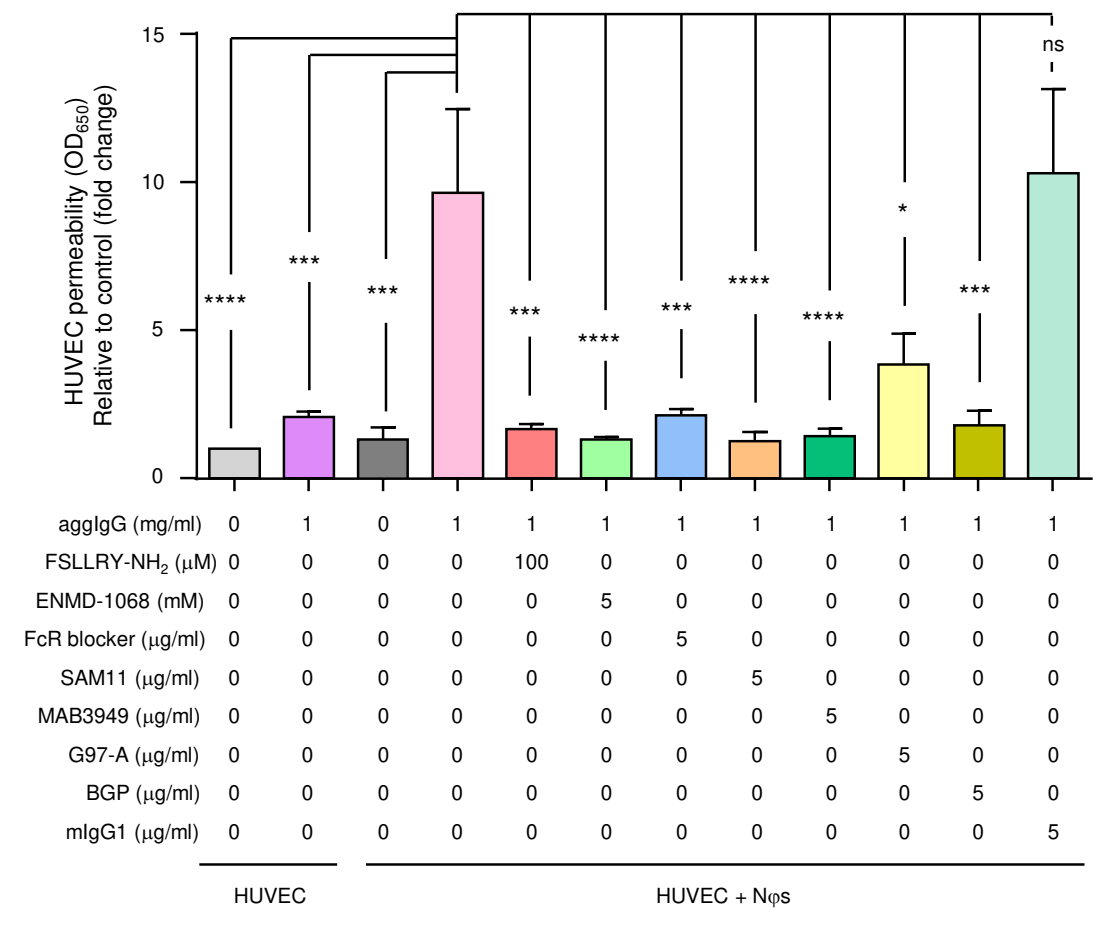


Fig. 7 GPR97-mediated PAR2 transactivation enhances $\mathrm{N} \varphi$-mediated bacterial phagocytosis and killing as well as endothelial cell activation and dysfunction. $a, b$. Phagocytosis (a) and killing (b) of live bacteria (E. coli, S. typhimurium, and S. aureus) by N $\varphi s$ incubated without or with GPR97 ${ }^{\mathrm{E}}-\mathrm{mFc}$. $\mathrm{mFc}$ was included as a negative control. Data are presented as means \pm SEM (one-way ANOVA). ${ }^{*} p<0.05 ;{ }^{* *} p<0.01$. c. The expressional analyses of cell activation markers (E-selectin, ICAM-1, VCAM-1 and eNOS) of HUVECs co-cultured with $\mathrm{N} \varphi s$ in the absence or presence of GPR97 ${ }^{\mathrm{E}}$-mFc. mFc was included as a negative control. HUVECs treated without or with LPS were used as a negative and a positive control, respectively. $n=3$ independent experiments. Data are presented as means \pm SEM (unpaired student's t-test). ${ }^{*} p<0.05 ;{ }^{*} p<0.01,{ }^{* * *} p<$ 0.001. d, e. Endothelial cell permeability assays of HUVECs co-cultured with N $\varphi s$. d. Assays were done in HUVEC-N $\varphi$ co-culture in the absence or presence of GPR97 ${ }^{\mathrm{E}}-\mathrm{mFc}$ at $37^{\circ} \mathrm{C}$ for $20 \mathrm{~h}$. mFc was included as a negative control. HUVECs alone treated without or with $\mathrm{mFc}$ were negative control groups, while those treated with thrombin were the positive control. $n=4$ independent experiments. e. The HUVEC-N $\varphi$ co-culture was treated without or with algGs in the absence or presence of protease inhibitors/PAR2 antagonists/blocking Abs as indicated. HUVECs alone treated without or with algGs were included as controls. $\mathrm{n}=3$ independent experiments. Data are presented as means \pm SEM (two-way ANOVA). ${ }^{*} p<0.05 ; * * p<0.01, * * * p<0.001, * * * * p<0.0001$; ns, non-significant. 


\section{Supplementary Files}

This is a list of supplementary files associated with this preprint. Click to download.

- Supplementaryinformation022022.pdf 